\title{
Kernos
}

Revue internationale et pluridisciplinaire de religion grecque antique

$27 \mid 2014$

Varia

\section{Le coureur et le guerrier}

Anthropologie de la course à pied et de ses vertus militaires

\section{Vincent Cuche}

\section{(2) OpenEdition}

1 Journals

\section{Édition électronique}

URL : http://journals.openedition.org/kernos/2269

DOI : $10.4000 /$ kernos.2269

ISSN : 2034-7871

Éditeur

Centre international d'étude de la religion grecque antique

\section{Édition imprimée}

Date de publication : 1 novembre 2014

Pagination : 9-50

ISBN : 978-2-87562-055-2

ISSN : 0776-3824

\section{Référence électronique}

Vincent Cuche, «Le coureur et le guerrier », Kernos [En ligne], 27 | 2014, mis en ligne le 01 octobre 2016, consulté le 20 avril 2019. URL : http://journals.openedition.org/kernos/2269 ; DOI : 10.4000/ kernos.2269

Ce document a été généré automatiquement le 20 avril 2019.

Kernos 


\section{Le coureur et le guerrier}

Anthropologie de la course à pied et de ses vertus militaires*

\section{Vincent Cuche}

1 À en croire la Charte olympique, dont le second des principes fondamentaux lie explicitement sport et pacifisme ; à en croire la Fondation internationale pour la trêve olympique, ou même l'ONU, qui émet avant chaque olympiade une résolution sur ce sujet ${ }^{1}$ , guerre et sport sont foncièrement et nécessairement antithétiques. Mais si la fondation pour la trêve olympique se réclame de l'exemple grec, les spécialistes du sport antique n'ont pas manqué de rappeler que cette trêve, dans l'Antiquité, n'avait nullement la portée qu'on voudrait aujourd'hui lui donner'2, et que les relations entre le domaine sportif et le domaine militaire demeuraient profondément ambiguës.

2 Pour faire bref, disons que, quoique l'agonistique sportive partage avec la guerre tout un ensemble de notions et de valeurs ${ }^{3}$, elle n'apparaît, dans son essence première, ni comme un dérivé de l'activité guerrière ni même comme une préparation militaire ${ }^{4}$; plus exactement, on préfère y voir deux domaines distincts où s'exprimaient de façon parallèle les valeurs aristocratiques, telles qu'elles se manifestent déjà dans les textes homériques. Au fur et à mesure que ces valeurs, élitistes et individualistes, perdaient de leur pertinence dans le monde des cités hoplitiques, où la guerre est une pratique fondamentalement collective, sont apparues des critiques de l'athlétisme fondées précisément sur le critère militaire. Des élégies de Tyrtée à l'Anacharsis de Lucien, toute une série de textes, tantôt comiques, tantôt sérieux, sont venus dénoncer, entre autres défauts, l'hyper-spécialisation de l'athlète, qui le rendait inapte à servir sa cité sur le champ de bataille 5 . Ou, pour le dire avec les mots d'Euripide, dans ce passage de l' Autolycos, drame satyrique fragmentaire ${ }^{6}$ :

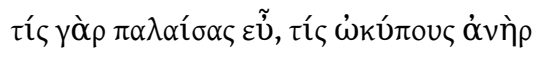

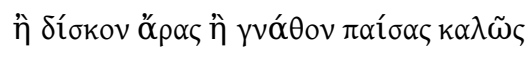

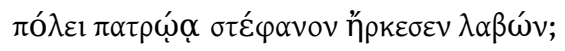

Qui a jamais défendu la cité de ses ancêtres avec une couronne gagnée en luttant, 
en l'emportant par ses pieds légers, en soulevant un disque ou en envoyant son poing en pleine mâchoire?

Évidemment, l'ambiguïté, la contradiction inhérentes aux rapports entre le sport, la cité et la guerre entrainaient nécessairement que la position inverse fût également soutenue (par exemple, au sein même de l'Anacharsis, par le personnage de Solon?). Et si l'on sort du domaine de l'argumentation polémique pour s'intéresser aux pratiques et aux représentations, on ne peut manquer de noter plusieurs indices de l'aura persistante qui accompagnait le champion sportif dans le domaine militaire: quand un sportif accomplissait quelques exploits à la guerre, les historiens comme Hérodote, Xénophon ou Diodore ne manquaient jamais de souligner sa qualité d'athlète ${ }^{8}$; à Sparte, les vainqueurs des concours stéphanites avaient le privilège de combattre à proximité immédiate du roi ; dans le même ordre d'idée, une coutume, dont on ne sait exactement si elle était répandue ou même historique, voulait que la procession triomphale qui accompagnait l'olympionice entrât dans la cité par une brèche faite dans le rempart, signifiant ainsi qu'une cité disposant de tels hommes n'avait nul besoin de murs ${ }^{9}$. Dans la plupart de ces exemples, les vertus de l'athlète, quoiqu'individuelles, étaient mises au service de la collectivité.

\section{Éloge de la course et pratiques hoplitiques}

4 Voilà pour les principes généraux ; il faut maintenant ajouter que tous les sports n'étaient pas égaux par rapport au critère guerrier; certains trouvaient grâce aux yeux des détracteurs de l'athlétisme. Prenons ainsi le livre VIII des Lois de Platon. Dans un passage où sont étudiées les règles qui régiront idéalement les compétitions athlétiques, l'interlocuteur athénien, dédaignant les concours de lutte, de pugilat et de pancrace, propose de les remplacer par des guerres en miniatures, avec utilisation de vraies armes: ces épreuves-là sauront bien, elles, préparer véritablement les citoyens à la guerre. Sont

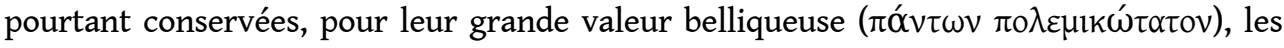
épreuves de courses traditionnelles, stade, diaulos, hippios, dolichos, - à cette différence qu'elles seront courues en armes, telles des hoplitodromies; Platon va jusqu'à introduire deux épreuves d'endurance: une course armée de soixante stades pour les hoplites, une autre de cent stades pour les archers ${ }^{10}$.

5 Pareille dichotomie entre les épreuves sportives lourdes, jugées inutiles à la guerre, et les épreuves de courses, valorisées pour leurs vertus militaires, se retrouve dans un passage frappant de l'Épaminondas de Cornelius Nepos. L'anecdote remonte à la période de la suprématie béotienne, entre 379 à 362 av. J.-C ; les Thébains étaient alors réputés pour leur supériorité physique. Selon Diodore et Plutarque, c'est parce que, encouragés par Épaminondas et Pélopidas, ils passaient leur temps dans les gymnases ${ }^{11}$. La version de Nepos est différente; l'Épaminondas qu'il nous présente condamne l'exercice de la palestre au profit de l'exercice des armes, tel un bon platonicien, méprise les épreuves de pure force, et ne retient des disciplines sportives que celles qui sont directement utiles à la guerre - c'est à dire, les épreuves de vitesse ${ }^{12}$.

On aurait tort de ne voir dans cette anecdote qu'un écho de plus des discours savants philosophiques et médicaux - qui condamnaient les épreuves dites lourdes ${ }^{13}$. Que la vitesse ait été prisée par Épaminondas et les Thébains trouve peut-être confirmation dans un témoignage contemporain, l'épitaphe de trois généraux béotiens morts à Leuctres. Cette dernière, au style assez contourné, se termine par le vers suivant ${ }^{14}$ : 


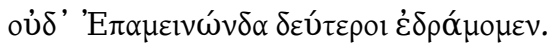

Nous n'avons pas couru moins vite qu'Épaminondas.

7 Énigmatiques par leur brièveté, ces quelques mots ont suscité plusieurs interprétations différentes; la plus naturelle consiste cependant à faire d'Épaminondas un parangon de vitesse, que les généraux défunts étaient fiers d'avoir égalé ; la rapidité à la course apparaît alors comme une pierre de touche de la valeur militaire ${ }^{15}$.

Comment expliquer cette exception en faveur de la course à pied ? En ce qui concerne Platon, il est évident que les compétitions de vitesse qu'il propose sont influencées par la présence de courses armées dans le programme des concours panhelléniques, depuis l'introduction de l'hoplitodromie à Olympie en 520 av. J.-C. ${ }^{16}$; cette course devenait ainsi la seule épreuve olympique à comporter un rappel explicite du monde militaire.

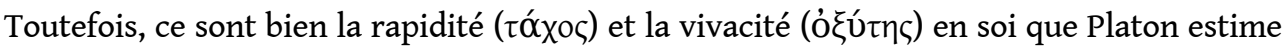
utiles à la guerre ${ }^{17}$; quant à Épaminondas, rien ne nous permet de dire que les épreuves de courses qu'il prônait aient été également des hoplitodromies. Et quand bien même elles l'auraient été, cela ne ferait que reculer le problème: pourquoi donc, parmi les concours panhelléniques, la course a-t-elle été la seule épreuve gymnique à se voir ainsi revêtir d'attributs guerriers? Il y a bien une étiologie de l'épreuve olympique de l'hoplitodromie, qu'on trouve dans le Gymnasticos de Philostrate, et qui relie son introduction à une guerre entre Élis et Dymé. Mais on n'accorde guère de valeur historique à cet aition postérieur de sept siècles à l'événement et dont le schéma ressemble trop à celui de plusieurs récits pseudo-historiques (qu'on examinera bientôt) ${ }^{18}$. Tenant davantage de la légende que de l'histoire, ce texte doit donc être réservé pour la suite de cette étude, et être étudié pour ses connotations légendaires plutôt que pour sa qualité documentaire. Délaissant Philostrate, les historiens en sont réduits à supposer que cette innovation olympique ne faisait que consacrer une épreuve déjà pratiquée localement ici et là ${ }^{19}$, ce qui, encore une fois, repousse le problème.

La pratique de l'hoplitodromie a le mérite de confirmer ce qu'on pouvait déduire des propos de Platon et des anecdotes autour d'Épaminondas : l'existence d'un lien intrinsèque entre la course à pied et la guerre ; pour autant, elle n'en constitue pas une explication. De fait, il peut paraître étonnant, voire paradoxal, qu'en pleine époque classique la course soit ainsi valorisée dans le domaine de la guerre : selon l'idéologie hoplitique, d'une grande constance depuis Tyrtée, la valeur de l'hoplite se définit selon une opposition entre le brave qui reste stable à son poste et le lâche qui s'enfuit, entre $\mu \varepsilon ́ v \varepsilon ı v$ et $\varphi \varepsilon u ́ \gamma \varepsilon ı v^{20}$. Or

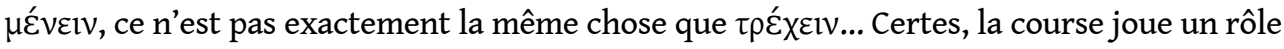
à deux moments de la bataille: au début, lorsque les deux armées se lançaient l'une contre l'autre, et à la fin, lors de la poursuite. Le terme $\delta \rho o ́ \mu$ o est d'ailleurs devenu un terme technique pour désigner le pas de course adopté durant la charge ${ }^{21}$. Cependant, ce pas de charge n'était pas véritablement un sprint ; et, d'autre part, ce n'était pas là que se décidait l'engagement. L'essentiel d'une bataille d'hoplites se déroulait ailleurs.

Dans le cas de la bataille de Leuctres, pour laquelle l'épitaphe des généraux béotiens vante leur rapidité ${ }^{22}$, on nous mentionne bien une charge de l'aile gauche thébaine ${ }^{23}$, et il serait tentant de la mettre en relation avec la tactique oblique adoptée par le stratège thébain : en concentrant le meilleur de ses troupes à l'aile gauche, en les disposant sur une profondeur inouie de cinquante rangs, Épaminondas entendait porter tout l'effort contre l'aile droite lacédémonienne, où se trouvaient les Spartiates et le roi Cléombrotos, prévoyant que leur défaite entraînerait celle du reste de l'armée ; en somme c'était en 
partie une arme psychologique. La charge pouvait avoir pour fonction de multiplier la violence du coup que la masse thébaine concentrée dans l'aile gauche infligeait aux Spartiates; de surcroît, il était de bonne tactique d'engager le combat le plus vite possible, alors que l'infanterie ennemie était encore embarrassée par la retraite désordonnée de sa cavalerie ${ }^{24}$. Tout cela est possible, mais il n'en demeure pas moins les sources anciennes sont unanimes sur ce point - que les Spartiates ont résisté très longtemps et que ce n'est qu'à la mort de cléombrotos que leurs rangs commencèrent à rompre $^{25}$; si l'on ajoute l'anecdote du "pas supplémentaire » demandé par Épaminondas à ses troupes pour assurer la victoire, on voit donc que la décision s'est faite de façon classique, au cours de la mêlée des deux lignes, lorsque chaque mètre de terrain se gagne au prix d'efforts extrêmes ${ }^{26}$. Il n'était pas alors question de courir. Si l'épitaphe des trois généraux morts à Leuctres a choisi de mettre l'accent sur leur vitesse, ce n'était sûrement pas dans le but de rappeler un moment précis et décisif de la bataille, et sans doute faut-il donner à cet $\varepsilon \dot{\delta} \rho \alpha{ }^{\mu} \mu \rho \mu \varepsilon v$ une valeur plus générale qu'il reste à explorer ${ }^{27}$.

Il en va de même avec la plus célèbre charge de l'histoire grecque, qui fut également la première selon Hérodote, celle de Marathon. Magnifiée par l'historien d'Halicarnasse, cette ruée des troupes athéniennes vers les contingents de Datis joua certainement un rôle considérable, tant sur le plan tactique - on minimisait ainsi le temps passé sous le feu des redoutables archers perses - que sur le plan psychologique: par cette charge quasi désespérée s'annulait le phobos que suscitait auparavant la seule vue des Perses ${ }^{28}$. Encore faut-il faire la part de l'exagération: la vraisemblance physiologique nous empêche d'accepter que les Athéniens aient vraiment couru les huit stades qui les séparaient du camp adverse en conservant leur ordre de bataille; tout au plus ont-ils pu parcourir le dernier stade au pas de course ${ }^{29}$. D'autre part, Hérodote prend soin de préciser que la bataille dura un long moment ${ }^{30}$; la course des hoplites athéniens, pour utile qu'elle fut, ne constitua pas l'élément décisif.

De tout cela il résulte que si, d'un point de vue pratique, la course a bien sa place à la guerre, on pourrait en accorder une tout aussi grande, sinon plus, à la boxe ou au pancrace, dont la pratique se rapproche davantage de celle de l'othismos, ce moment de mêlée décisif où les deux camps poussent et luttent à portée de bras, bouclier contre bouclier (rapprochement qu'effectue d'ailleurs Plutarque, dans un de ses Propos de table ${ }^{31}$ ). Les seules considérations tactiques ne sauraient donc suffire à expliquer pourquoi la course parait jouir d'une aura militaire privilégiée.

Il faut alors recourir à d'autres types d'explications; examiner les connotations de la course, les contextes dans lesquels on la pratique, les faits religieux et les discours mythiques qui l'entourent ; bref, esquisser une anthropologie de l'acte de courir.

\section{Significations rituelles}

Tout discours sur la course en tant qu'épreuve sportive devrait commencer avec le prestige particulier dont elle jouissait. Comme le résume Xénophane dans son couplet

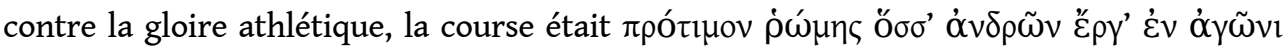
$\pi \varepsilon ́ \lambda \varepsilon l$, « la plus honorée des compétitions de force parmi les hommes » ${ }^{32}$, et cela avant tout en raison de sa primauté olympique. Plus ancien concours du programme olympique, le stade en constitua pendant longtemps l'unique épreuve et donnait à son vainqueur l'éponymie de l'olympiade; et lorsqu'on voulut enrichir le programme olympique, les deux premiers concours à être rajoutés furent le diaulos et le dolichos, soit deux autres 
épreuves de course ${ }^{33}$. La plupart des concours chrématites semble avoir respecté cette primauté du stade, qui peut donc revendiquer, mutatis mutandis, le statut qu'occupe de nos jours le cent mètres; on sait par exemple qu'aux Panathénées athéniennes, le vainqueur du stade était le mieux loti des champions gymniques, avec ses quatre-vingt amphores pour les hommes, soixante pour les jeunes et cinquante pour les enfants ${ }^{34}$. Et si les épreuves lourdes, et notamment le pancrace, ont pu gagner en popularité durant l'époque hellénistique et romaine ${ }^{35}$, cela ne signifiait en rien une dévalorisation de la course : les inscriptions honorant les athlètes témoignent au contraire d'une popularité durable des épreuves de vitesse, tandis que de nouvelles épreuves et compétitions, comme l'hoplitodromie des Eleutheria, ou les prestigieux Capitolia (pour lesquels Domitien construisit le stade de la place Navone) leur assuraient une place privilégiée ${ }^{36}$.

Étant donné que les compétitions homériques comportaient déjà un large éventail de concours (course, saut, lancer, boxe, lutte, épreuves hippiques), la pauvreté du programme olympique originel et la position solitaire de la stadiodromie ont logiquement suscité quelques interrogations - d'autant que la chronologie des premiers temps olympiques ne repose que sur les travaux d'un érudit du $\mathrm{IV}^{\mathrm{e}}$ siècle, Hippias d'élis ${ }^{37}$. Mais comme le souligne Hugh Lee, à la différence des concours funéraires pour Patrocle ou des récréations sportives des Phéaciens, les Olympia étaient avant tout une fête religieuse en l'honneur de Zeus. Or, s'il est une épreuve sportive qui a clairement occupé une place rituelle au sein des célébrations grecques, c'est bien la course ${ }^{38}$. Présente dans de nombreuses fêtes, même dépourvues de concours gymniques, prenant souvent la forme d'une lampadédromie, la course était d'autant plus intégrée à l'action religieuse qu'elle était censée s'achever à l'autel de la divinité honorée ; c'est du moins le fonctionnement habituel de la lampadédromie, dont le vainqueur allumait de son flambeau le feu sacré de l'autel ${ }^{39}$; et c'est également l'aition du stade et du diaulos, tel que le rapporte Philostrate. Il vaut la peine de le reproduire dans son intégralité ${ }^{40}$ :

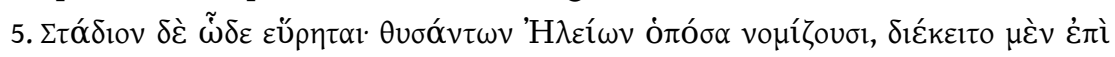

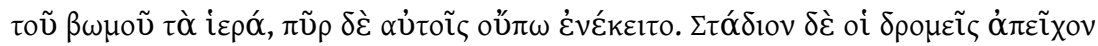

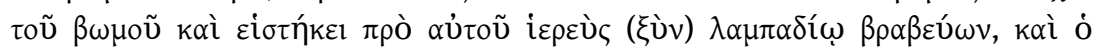

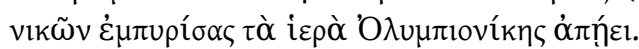

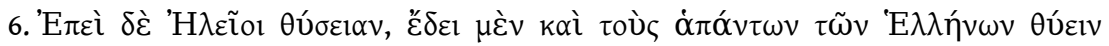

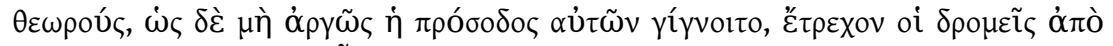

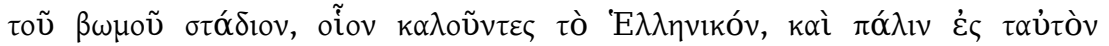

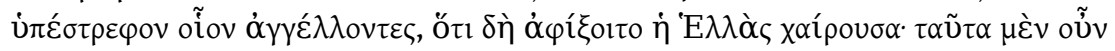

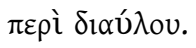

5. Voici comme a été inventé le stade. Quand les Éléens avaient sacrifié selon leur coutume, les parts sacrificielles étaient disposées sur l'autel mais le feu n'y était pas encore mis. Les coureurs se tenaient à un stade de distance de l'autel, devant lequel se tenait le prêtre avec un flambeau, qui servait d'arbitre; le vainqueur mettait le feu aux parts sacrificielles et s'en retournait avec le titre d'olympionice.

6. Quand les Éléens avaient sacrifié, il fallait aussi que tous les théores de Grèce sacrifiassent à leur tour, et pour ne pas retarder leur procession, les coureurs partaient de l'autel en courant sur la longueur d'un stade, comme pour inviter tout le monde grec, puis revenaient à leur point de départ comme pour annoncer que la Grèce était arrivée et apporter son salut. Voilà donc pour l'épreuve du diaulos.

C'est à partir de ces éléments que Walter Burkert a tenté de retrouver, dans une analyse demeurée célèbre, le sens religieux originel des concours olympiques. Rappelant que la fête comportait le sacrifice nocturne d'un bélier noir au héros Pélops, dont le bothros se 
trouvait à l'ouest de l'Altis, ainsi que le sacrifice diurne d'un taureau sur le gigantesque autel de cendre de Zeus, un peu plus à l'est, et considérant que le stade primitif devait aboutir à l'autel de cendre, il a proposé de voir dans la course une action rituelle à part entière, reliant les deux lieux sacrificiels, et signifiant le passage de l'obscurité à la lumière, de la mort sanglante à la purification par le feu et à la vie, via la manifestation de force du coureur ${ }^{41}$.

En intégrant également le mythe du démembrement de Pélops à cet ensemble, Burkert pouvait rattacher les Olympia à sa théorie générale du sacrifice comme acte violent empreint de mauvaise conscience, accompagné d'une "comédie de l'innocence » et culminant en une restauration joyeuse des forces vitales. Mieux : certaines particularités propres au Pélops d'Olympie lui paraissaient ressortir au même schéma que celui qu'il avait déduit de son analyse des rites et mythes du mont Lycée - histoires de cannibalisme, de culpabilité et d'expiation, d'initiations et de réintégrations ${ }^{42}$. Ce faisant, il retrouvait là une théorie de la fonction initiatique des concours olympiques déjà défendue avec force par Henri Jeanmaire, mais qui, pour le savant allemand, restait sousjacente, l'initiation ayant été progressivement rejetée à l'arrière-plan par l'évolution des concours $^{43}$. À tout le moins, le mois d'entraînement et d'abstinence imposé aux athlètes pouvait évoquer le temps de marginalité des rites initiatiques, les festins célébrant la fin des concours et la fin de la trêve permettant ensuite aux sportifs de réintégrer le temps ordinaire $^{44}$.

Inévitablement conjecturale, cette reconstruction du sens primitif de la course olympique n'a pas manqué de susciter quelques remises en cause; les critiques se sont portées principalement sur l'emplacement du stade primitif et sur l'ancienneté du lien des concours avec le mythe de Pélops. Ainsi, les explorations archéologiques d'Alfred Mallwitz ont démontré que le stade n'avait pas changé de place au moins depuis le début du vII ${ }^{e}$ siècle, ce qui, sans l'interdire totalement, fragilise l'idée que la course primitive aboutît à l'autel de Zeus ; selon le même savant, le Pélopion ne serait pas antérieur à 700 av. J.-C ${ }^{45}$. Encore une fois, il peut être dangereux de prendre Philostrate pour un historien... Ce n'est pas que son aition soit sans valeur, bien au contraire : il en dit long sur l'imaginaire attaché à cette épreuve sportive; mais ce n'est pas un document sur le premier siècle de l'olympisme, dont il faut bien admettre qu'on ne sait pas grand chose.

La question initiatique peut, elle aussi, être soumise à la critique, d'autant que son application dans le domaine des sciences des religions antiques, voire le concept même d'initiation, ont été fortement remis en cause ces dernières années ${ }^{46}$. En ce qui concerne la course, cependant, nous sommes dans un domaine plus solide. Certes, on ne saurait démontrer que tous les concours de course à pied occupaient la fonction d'un rite de passage; du moins convient-il de rappeler la fréquence des courses d'éphèbes dans le programme des fêtes civiques grecques, et le lien étroit de cette classe d'âge avec le dromos, une piste qui, dans bien des cités, était située sur l'agora ou à proximité immédiate ${ }^{47}$. C'est notamment le cas à Sparte, où, le dromos servait de cadre à plusieurs actes rituels ; selon Pausanias, les jeunes venaient s'y s'entraîner à la course, et lorsqu'ils quittaient le statut éphébique pour entrer dans la première étape de l'âge adulte, ils y sacrifiaient au dieu patron du dromos, Héraclès ${ }^{48}$. C'est probablement sur cette même piste que se déroulaient des courses de jeunes filles ${ }^{49}$ ainsi que la staphylodromie, une poursuite rituelle à laquelle prenaient part de jeunes célibataires lors de la grande fête des Carneia. Cette dernière, souvent analysée comme le terme de l'agôgé spartiate, était en effet consacrée à un dieu - Apollon Carneios - logé tout près du dromos, et comprenait 
en outre des compétitions gymniques dont le programme donnait la prépondérance aux épreuves de courses ${ }^{50}$. Enfin, le cas le plus explicite par son vocabulaire provient de Crète - pays propice à la course, selon le Platon des Lois ${ }^{51}$-, où les jeunes étaient dits apodromoi et devaient attendre la fin de leur agela pour prétendre au titre de dromeus, qui désignait

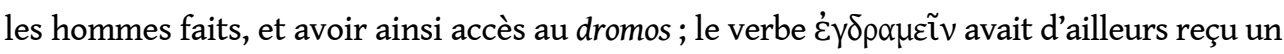
sens technique dénotant le rite de fin d'initiation ${ }^{52}$.

Les dossiers spartiates et crétois sont d'autant plus précieux qu'ils constituent sans doute les exemples les plus nets et les moins contestés de rites de passages en Grèce ancienne ${ }^{53}$. Mis bout à bout, ils ne laissent subsister guère de doutes sur l'existence d'un lien consubstantiel entre la course à pied et les initiations de classes d'âge en pays dorien. Doit-on voir dans ces rites éphébiques la clef du symbolisme de la course dans le domaine guerrier? Je dirai plutôt qu'il faut retourner le raisonnement. On l'a souvent montré, les valeurs éphébiques sont des valeurs inverses de celles du citoyen-soldat, et toute une série de rites, notamment de travestissement, mettent en scène l'inversion que constitue ce passage. Mais les exemples dont nous disposons semblent bien faire de la course un rite de sortie, que ce soit aux Oschophories athéniennes, aux Carneia doriennes et plus encore en Crète où c'est bien l'homme adulte qui est appelé dromeus, et non l'éphèbe ${ }^{54}$. Dans ce contexte, si la course fait office de frontière entre monde adolescent et monde adulte, guerrier, c'est à ce dernier que ses valeurs appartiennent. J'en trouve confirmation dans le nom d'un rite macédonien, les diadromai, consistant en des courses et des simulacres de combats, et dont la fin, semble-t-il, était d'intégrer à l'armée les jeunes gens qui avaient déjà terminé leur éducation ${ }^{55}$. La conclusion s'impose : l'usage de la course comme rite de passage tient à ses connotations viriles ${ }^{56}$ et guerrières, et non l'inverse.

21 Au final, ce premier examen des contextes religieux de la course nous permet de confirmer le lien qu'elle entretient avec le monde de l'homme fait et du guerrier, sans toutefois l'expliquer véritablement. On notera également que, dans les pratiques sociales comme dans le discours étiologique d'un Philostrate, elle apparaît comme un acte empreint de ritualité - donc un acte efficace per se - et chargé d'une certaine antiquité. Une course pouvait modifier le statut d'un coureur ; une victoire olympique à la course pouvait même lui obtenir le statut de héros cultuel : c'est ce que vont montrer les exemples suivants.

\section{Coroibos, Orrhippos et Oibotas : trois coureurs cultuels}

Du tout premier vainqueur olympique, nous ne savons pas grand chose. Nommé Coroibos, d'ethnique éléenne, il n'avait pas obtenu de statue dans l'enceinte olympique - la coutume n'en existant pas encore ${ }^{57}$. Des quelques bribes d'informations que nous fournissent de tardifs antiquaires, nous apprenons qu'il était également mageiros et que son tombeau se trouvait à la frontière entre l'Élide et l'Arcadie, à proximité de la cité d'Héraia ${ }^{58}$. Le premier point a attiré l'attention des sociologues du sport. Plutôt que voir en Coroibos un boucher professionnel de basse extraction, on a conclu qu'il fallait vraisemblablement donner à ce titre de mageiros toute sa signification rituelle de sacrificateur; du même coup le premier coureur olympique était rattaché à la sphère du sacrifice, déjà mise en avant par l'aition de Philostrate ${ }^{59}$. Quant à sa tombe frontalière, elle ne peut manquer d'évoquer ces sanctuaires de héros ou de dieux qui définissent les territoires civiques par leur position aux confins ${ }^{60}$. 

déduit que de leurs biographies et de la position de leurs tombes, les choses sont plus nettes pour le cas d'Oibotas de Paleia. Vainqueur à la sixième Olympiade (756 av. J-C.), ce coureur fut le premier Achéen à remporter le stade, la compétition ayant jusqu'alors été dominée par les athlètes d'Élide et de Messénie ${ }^{65}$. Pas plus que ses prédécesseurs il n'avait été représenté en ronde-bosse, que ce soit à Olympie ou dans sa patrie, puisque ces pratiques n'apparurent que bien plus tard. Réinterprétée à la lumière de l'explosion 
statuaire qui caractérisa le sport grec à partir de la fin de l'archaïsme, cette absence fut sans doute le point de départ de la légende d'Oibotas. L'histoire se trouve dans la Périégèse : courroucé de n'avoir reçu aucun honneur de la part de ses concitoyens, l'olympionice aurait proféré une malédiction qui empêcha toute victoire achéenne pendant trois siècles. Quelques temps avant la quatre-vingtième olympiade (460 av. J.-C.), à la suite d'un oracle delphique, les Achéens se résolurent à consacrer une statue de l'athlète à Olympie, accompagnée d'une épigramme, et, délivrés ipso facto de la malédiction, remportèrent la victoire au stade en la personne de Sostratos de Pallène. «Encore de mes jours, conclut Pausanias, les Achéens qui se disposent à concourir aux Olympia ont coutume d'offrir des

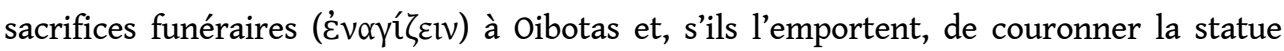
d'Oibotas à Olympie ${ }^{66}$. Indice sans équivoque d'un culte, ces enagismata étaient très probablement effectués sur le monument funéraire de l'athlète, situé à Paleia, dans la chôra de Dymé ; c'est d'ailleurs lors de son passage à Dymé que Pausanias nous rapporte cette histoire, qu'il devait tenir d'exégètes locaux ${ }^{67}$.

Si l'on peut ranger l'histoire d'Oibotas au rang de légende, c'est parce qu'elle semble avoir pour principale fonction de justifier la confection a posteriori d'une statue pour un athlète disparu depuis des siècles; et, surtout, parce que cette terrible malédiction d'Oibotas n'est que pure invention. Entre l'olympiade d'Oibotas et celle de Sostratos, ce ne sont pas moins de trois Achéens qui remportèrent la couronne d'olivier: Icaros d'Hyperésia, vainqueur du stade en 688 , Phanas de Pellène, vainqueur le même jour du stade, du diaule et de la course armée, en 512 , ce qui fit de lui le premier triastès de l'histoire ${ }^{68}$, et Pataicos de Dymé, pour l'épreuve éphémère du trot, en $496^{69}$. Cette dernière victoire nous fournit d'ailleurs un terminus post quem pour l'invention du motif de la malédiction, qu'on peut tenter de croiser avec le terminus ante quem que constitue la victoire de Sostratos ${ }^{70}$.

Puisque les Achéens avaient honoré rétroactivement leur proto-olympionice par une statue olympique, le Périégète avait pu la mentionner lors de sa visite de l'Altis; il rapporte à cette occasion un curieux détail supplémentaire de la légende d'Oibotas : selon les Achéens, l'athlète aurait combattu dans les rangs des Grecs à la bataille de Platées ${ }^{71}$. Perplexe devant cet anachronisme évident (d'autant que, d'après Hérodote, aucun Achéen n'avait participé à cette bataille $\mathrm{e}^{72}$ ), Pausanias fait le choix de refuser en bloc cette tradition. Il y aurait pourtant un moyen de la rendre acceptable : en faire une épiphanie héroïque, comme on en connaît tant pour les guerres médiques ${ }^{73}$. Une apparition miraculeuse du héros Oibotas en 479 av. J.-C. concorderait parfaitement avec la date de l'érection de sa statue, qu'il faut vraisemblablement situer entre 496 et 460 . Sans doute doit-on situer l'héroïsation d'Orrhippos dans la même période, puisque l'épigramme qui fut gravée sur son tombeau est attribuée à Simonide ${ }^{74}$.

Les héroïsations tardives de ces coureurs des premiers temps de l'olympisme permettent de les rattacher à tout un groupe d'olympionices, parfaitement historiques et bien mieux connus, qui reçurent des honneurs divins ou héroïques entre la fin $\mathrm{du} \mathrm{vi}^{\mathrm{e}}$ et la première moitié du ve siècle $\mathrm{e}^{75}$. Pour la plupart de ces personnages, le culte s'est accompagné de quelques traits biographiques tenant davantage de la légende que de l'histoire, et organisés suivant un schéma récurrent, qu'on peut résumer ainsi: d'une force surhumaine qui se manifeste par des exploits spectaculaires, l'athlète remporte la victoire olympique mais, victime d'une injustice de la part des hellanodices ou de ses concitoyens, il ne reçoit pas les honneurs dus. Sa colère provoque une catastrophe pour sa cité, et luimême ou sa statue disparait de manière mystérieuse; la résolution de la crise passe, comme de juste, par un oracle delphique et par l'instauration d'un culte qui apaise enfin 
la colère du nouveau dieu ou héros. À cela peuvent s'ajouter des motifs secondaires, tels que la paternité divine de l'athlète ou les pouvoirs miraculeux de sa statue ${ }^{76}$.

On voit combien facilement l'histoire d'Oibotas se fond dans ce schéma : privé d'honneurs - c'est-à-dire, sans doute, de sa statue - l'athlète exerce sa vengeance dans le domaine olympique non seulement sur sa cité, mais sur toute l'Achaïe, jusqu'à ce qu'un oracle et un culte viennent concilier le héros et inverser son action : à l'absence de statue, facteur de malédiction, s'oppose dès lors la présence bénéfique de la statue olympique, que les Achéens couronnaient à chaque nouvelle victoire ${ }^{77}$. Bref, tout porte à croire qu'entre 496 et 460 , Oibotas obtint non seulement une statue et un culte mais également une biographie, et que cette dernière fut confectionnée sur le même modèle que celui qui servit à mythifier la vie et les exploits d'authentiques champions du début de l'époque classique.

31 De fait, si l'on retrouve ce schéma pour d'autres athlètes d'autres époques, ce n'est qu'entre la fin du $\mathrm{vI}^{\mathrm{e}}$ siècle et la fin du $\mathrm{v}^{\mathrm{e}}$ qu'il entraîne la création d'honneurs cultuels. Pour tenter de rendre compte de cette synchronie, plusieurs savants ont insisté sur l'inscription historique de ces nouveaux cultes ${ }^{78}: c^{\prime}$ est l'époque des grandes épinicies, un âge d'or de l'agonistique grecque, qui coöncide avec une période de transition et de modification des équilibres socio-politiques au sein des cités grecques. La disparition progressive des grandes tyrannies $\mathrm{du} \mathrm{VI}^{\mathrm{e}}$ siècle, les changements militaires consécutifs aux guerres médiques, l'extension du modèle démocratique s'unissent pour créer un contexte civique en mouvement perpétuel, dans lequel le rapport de la cité à ses élites devient extrêmement ambigu. Issu de familles renommées, faisant lui-même partie de ces élites et incarnant par son succès un charisme exceptionnel, le champion sportif exprime dans le domaine sportif une aretè qui serait potentiellement dangereuse si elle s'exprimait dans les domaines militaires ou politiques. L'aura de victoire qui l'entoure, ou kudos, doit être réintégrée dans la cité à travers ce que Leslie Kurke a appelé un traffic in praise sous peine de rupture d'équilibre ${ }^{79}$; si ce n'est pas le cas, soit que le kudos soit surabondant, soit que les honneurs adéquats ne lui soient pas rendus, l'instauration du culte parait le moyen idoine à rétablir le fonctionnement de cette économie du $k u d o s^{80}$, tenant à la fois l'athlète charismatique à distance de la communauté, rendant justice à son charisme, mais permettant également à la cité de s'accaparer son kudos sans danger pour ellemême. Ce dernier point nous permet également de rajouter un facteur déclenchant essentiel à ces phénomènes de déification ou héroïsation: ils interviennent souvent, semble-t-il, au moment où leur cité, en situation de crise, éprouve le besoin de se parer d'une figure charismatique et de récupérer son aura de victoire pour surmonter l'épreuve ${ }^{81}$. De par sa carrière militaire et son rôle territorial, de par son voisinage également avec le héros Coroibos, l'olympionice Orrhippos a pu apporter sa protection à Mégare à une époque où Corinthe lui contestait des régions frontalières, dans le deuxième quart $d u v^{e}$ siècle $^{82}$. Quant à Oibotas, dont le culte est explicitement pan-achéen, j'ai suggéré ailleurs d'inscrire son héroïsation dans le double cadre de la formation du koinon achéen et de la seconde guerre médique : l'absence des Achéens à la bataille de Platées (ou leur retard, selon la version plus clémente de Pausanias) avait été remarquée ${ }^{83}$ et, à l'heure où les vainqueurs gravaient fièrement leur nom sur la colonne delphique et discutaient du sort des médisants, il fallait bien une sorte de deus ex machina qui vînt résoudre cette situation de crise. L'épiphanie du nouvel héros Oibotas compensait ainsi l'absence collective des Achéens ${ }^{84}$. 

que les cités de Grande-Grèce s'étaient massivement abstenues lors de la seconde guerre médique, un athlète de Crotone, Phayllos, triple pythionice au pentathlon et au stade, avait armé à ses propres frais un navire et rejoint la flotte grecque à Salamine; ses exploits sportifs et militaires figuraient d'ailleurs conjointement sur la dédicace accompagnant l'offrande qu'il déposa sur l'Acropole athénienne. Si dans l'immédiat cet acte individuel ne suffit pas à faire graver le nom de Crotone sur la colonne de Platées, l'aura de Phayllos, par une saine économie du kudos, rejaillit sur sa patrie, rapidement associée à son haut fait par la postéritée ${ }^{85}$. Phayllos de Crotone) semblent avoir été sollicités en des périodes de conflits armés où l'on comptait sur leurs vertus victorieuses. On voit mal, toutefois, ce que leur qualité de sprinteur pouvait leur apportait de spécifique en cette occasion ${ }^{86}$. Aussi s'agit-il sans doute d'une limitation du schéma d'analyse que nous avons suivi jusqu'ici, qui articule une prédisposition générale des athlètes à l'héroïsation (en raison de l'aretè investie avec succès dans les concours, et qui fait de l'athlète un personnage à la fois charismatique et quelque peu tabou, répandant tour à tour prestige et fléau sur sa cité) avec des facteurs déclencheurs historiquement spécifiés (c'est à dire une situation politique où la communauté éprouve le besoin de récupérer ce kudos athlétique tout en maintenant l'athlète à distance). Car, à parler de la sorte, on court le risque de passer à côté de ce qui est spécifiquement athlétique dans ce processus d'héroïsation ${ }^{87}$; en particulier, on ne rend pas compte de la position inégale des différentes disciplines olympiques. Or, malgré la popularité bien connue des compétitions hippiques, et particulièrement de la course de chars, première épreuve des Olympia, malgré le faste que lui ont apporté les épinicies de Pindare et de Bacchylide, on ne connaît aucun vainqueur au quadrige, ou de quelconque épreuve hippique, qui ait fait l'objet d'un culte ${ }^{88}$. Ce dernier ne semble donc se concevoir que par l'intermédiaire du corps de l'athlète, dans le cadre d'un effort brut, voire excessif. Et de fait, sur la petite douzaine d'athlètes héroïsés ou divinisés que l'on connaît, la plupart proviennent de deux disciplines lourdes, le pugilat et le pancrace ${ }^{89}$. Les coureurs ne sont que deux - trois si l'on rajoute Coroibos d'élis - et pour chacun d'entre eux il s'agit de l'héroïsation tardive d'un athlète remontant aux premiers temps de l'athlétisme et lui-même novateur (premier coureur, premier vainqueur achéen, premier coureur nu ${ }^{90}$ ).

Pourquoi un tel décalage chronologique pour les coureurs seulement? Pour les cités et ethnè qui ne disposaient pas d'autre olympionice à vénérer (ce qui n'était pas le cas des Achéens, ni même de Dymé), nous pouvons supposer qu'il était pratique d'avoir recours à des athlètes oubliés dont on pouvait aisément réécrire l'histoire, l'absence de statue olympique à cette époque fournissant alors le motif des honneurs oubliés. Dès lors qu'on recherchait un athlète des premières olympiades, on ne pouvait avoir affaire qu'à un sprinteur. Je présenterai pourtant deux objections à cette explication. La lutte est apparue dès la dix-huitième olympiade (708 av. J-C.) et le pugilat à la vingt-quatrième (688 av. J.-C.), soit une génération seulement après la victoire d'Orrhippos. Il était sûrement possible de répéter la même opération héroïsante avec les premiers vainqueurs des épreuves lourdes, guère plus connus qu'Oibotas ou Orrhippos ; on le ne fit pas. D'autre part, cela n'explique pas qu'aucun coureur d'époque classique n'ait fait l'objet d'un culte à une époque où l'on en accordait à ses collègues pugilistes et pancratiastes. À chaque fois qu'on a honoré un coureur, il a semblé nécessaire de le renvoyer dans des temps 
primitifs, comme si les valeurs connotées par la course avaient besoin de la caution d'une grande antiquité et ne pouvaient trouver place sans médiation dans l'idéologie de l'époque classique. Nous retrouvons là des conclusions déjà atteintes précédemment.

Autre question: qu'a de commun la course à pied avec des épreuves dont elle semble l'antithèse, épreuves lourdes où les arbitres veillent à ce qu'on ne s'enfuie pas et qu'on attende l'adversaire de pied ferme ${ }^{91}$ ? Que le pugilat et le pancrace aient particulièrement contribué à donner un statut cultuel à ceux qui les pratiquaient, au fond, cela se conçoit assez bien. Il s'agit là de sports où la violence était acceptée, où les coups et les blessures pouvaient entraîner jusqu'à la mort de l'adversaire ${ }^{92}$ - en somme, quasiment une épreuve

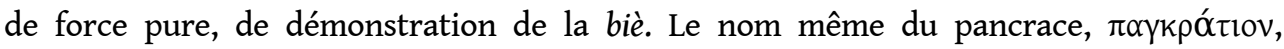
désigne bien la «supériorité complète » du vainqueur de l'épreuve. À partir des vies de Cléomèdès, d'Euthymos, de Théogénès ou de Pouludamas, on décèle aisément le modèle qui court en filigrane derrière ces champions : Héraclès, la Biè Heracleiè, l'inventeur des concours olympiques, que les sources mythologiques nous montrent souvent victorieux du pancrace ${ }^{93}$. La chose est désormais bien étudiée: il existe tout un réseau de représentations autour des notions de force pure, de violence meurtrière, qui associe les champions de boxe et de pancrace à l'Alcide et à quelques autres figures, leur conférant une sacralité aux connotations autant positives que négatives ${ }^{94}$. Que vient faire la course à pied dans ce contexte?

Pour répondre à ces questions, j'a-urais à nouveau recours à la notion de modèle mythologique : de même que la figure d'Héraclès semble être le fil conducteur du portrait de certains boxeurs et pancratistes, derrière le coureur, que ce soit à la guerre ou à Olympie, il est difficile de ne pas évoquer Achille aux pieds légers. À étudier ce que signifient ces pieds légers et la notion de vitesse dans les poèmes homériques, on voir se déployer bien des connotations attachées à l'imaginaire de la course.

\section{Courir et voler dans les poèmes homériques}

Assurément, il n'y a rien d'original à voir en Achille un modèle des champions de course à pied $^{95}$; bien avant nous, Pausanias avait avancé cette hypothèse en racontant les exploits de Théogénès. Le fameux champion thasien, participant aux concours de Phthia, en Thessalie, avais commis l'originalité de négliger le pugilat et le pancrace, ses disciplines habituelles, pour courir - et remporter ! - le dolichos, la course de fond d'une vingtaine de stades. Pausanias tient une explication toute prête, que j'adopterais volontiers: " c'était, je crois, par émulation envers Achille qu'il souhaitait remporter une victoire à la course dans la patrie du plus rapide de ceux qu'on appelle les héros ${ }^{96}$.

À Olympie également, le Péléide demeure un coureur : avec Héraclès, il patronne le dromos d'Élis près duquel se trouvait son cénotaphe; les femmes éléennes y venaient effectuer des lamentations rituelles la veille des Olympia. En cet Achille au dromos se renouent fil athlétique et fil initiatique, comme le montre également le sacrificeque les éphèbes spartiates lui offraient avant le combat du Platanistas ${ }^{97}$.

Contemplons donc Achille, tout à la fois le plus fort et le plus rapide des Achéens. Ce

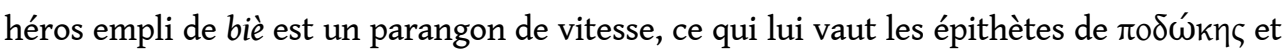

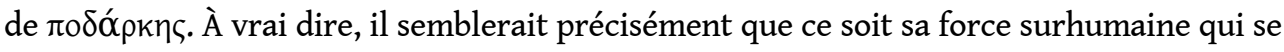

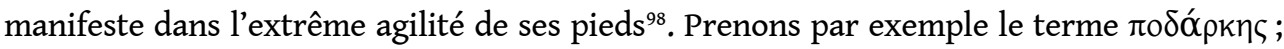
il n'est pas fréquent ; aussi vaut-il la peine de rappeler, après Gregory Nagy, que c'est le 
nom du fils d'Iphiclos, autre prince thessalien à la rapidité légendaire et qui a droit à

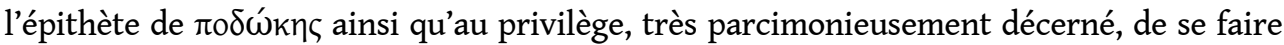
appeler Biè Iphicleie ${ }^{99}$. Autre exemple homérique, Areithoos, héros béotien de la génération d'avant Nestor, contracte en son nom même, "rapide par Arès ", les valeurs de la vitesse et de la guerre. On n'en regrette que davantage de ne pas connaître les exploits qui justifiaient ce nom ; tout ce qu'on en peut dire, c'est que, combattant primitif, armé de sa lourde massue de fer, il se révèle être, selon l'expression de Nagy, un de ces héros de biè, un représentant de la force ${ }^{100}$. En somme, pour Achille et pour ces héros préiliadiques, la puissance physique se distribue indifféremment dans la rapidité comme dans la valeur guerrière ${ }^{101}$.

Sans doute Gregory Nagy a-t-il raison de voir là un héritage indo-européen; derrière Achille, Iphiclos et Areithoos transparaissent les figures indiennes de Vayu, dieu vent, patron des compagnies de guerriers, et surtout de Bhima, héros plein de force, voire de brutalité, et rapide comme les vents ${ }^{102}$. Reste à déterminer ce qui est resté de cet héritage, et les inflexions qu'il a subies au cours d'un millénaire de guerres grecques et de concours olympiques. L'association de la force aux vents, par exemple, s'est faite plus discrète mais peut encore parfaitement se lire dans l'Iliade : ces éléments sont eux aussi dotés d'une biè

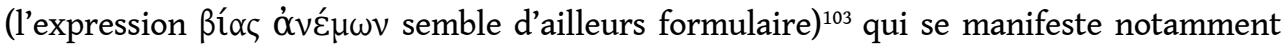
par leur rapidité. Transmise à leur progéniture, cette puissance véloce permet d'établir tout un réseau d'équivalence entre des créatures relevant de règnes distincts et dont la fabuleuse rapidité s'exprime par des images comparables. Ainsi Zéphyr, le plus vite d'entre les vents, s'est uni à la Harpye Podargé pour engendrer les invincibles chevaux d'Achille, Zanthos et Balios ${ }^{104}$. De Borée également sont nés des chevaux, lorsque le dieu se prit d'amour pour les juments d'Érichthonios : leurs rejetons pouvaient galoper sur la pointe des flots et, sur terre, effleuraient à peine le sommet des épis de blé ${ }^{105}$. Autres enfants de Borée, Zétès et Calaïs, qu'on appelle communément les Boréades, étaient dotés d'ailes, et c'est sans surprise qu'on apprend qu'ils avaient remporté les épreuves de course à plusieurs concours ${ }^{106}$.

41 Il est ainsi possible de déterminer un ensemble de figures épiques qui partagent quelques caractéristiques bien particulières, dessinant une espèce d'archétype de la rapidité personnifiée : un lien avec la force brute, quasi cosmique, que décrivent les termes ßín et is ; des pieds agiles ou légers; le port d'ailes aux chevilles, au dos ou aux tempes ${ }^{107}$; une course si légère qu'elle permet de glisser sur la terre comme sur la mer sans laisser de traces et que désigne généralement le verbe $\theta \varepsilon ́ \varepsilon ı v$ et ses dérivés ${ }^{108}$. Ces figures sont les Vents, les chevaux par eux engendrés ${ }^{109}$, d'autres figures divines telles qu'Iris, dont les épithètes sont podénémos, "aux pieds de vent " ou aellopos, "aux pieds de tempête " "10, parfois même des navires ${ }^{111}$, et, parmi les humains, des héros exceptionnels tels les Boréades, les Argonautes Iphiclos et Euphémos ${ }^{112}$, et bien sûr Achille, qui, de surcroit, possède les chevaux les plus rapides du camp achéen ${ }^{113}$.

Entre tous ces parangons de rapidité, les jeux de comparaisons, de substitution et de métaphores abondent, dans un incessant renchérissement de la tradition épique sur son modèle initial. Pour Apollonios de Rhodes, c'est Euphémos de Sparte qui tient le rôle du héros aux pieds légers ${ }^{114}$; pratiquant cette " course sur les pointes » qui lui permet de

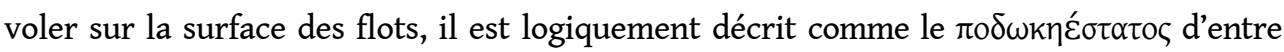
tous les Argonautes - un rôle que d'autres traditions attribuaient à Iphiclos. Mais dans ce poème où la rapidité est un trait partagé par bien des héros, et jusqu'au navire lui-même ${ }^{115}$, l'exemple le plus riche en images est peut-être fourni par l'épisode des Harpyes, qui 
concentre dans un saisissant concours de vitesse la plupart des figures et des thèmes évoqués ${ }^{116}$. Entre ces monstres génitrices de chevaux, dont la rapidité égale celle de l'éclair ou dépasse les bourrasques de Zéphyr, les fils de Borée, qui se ruent à leur poursuite, tels des chiens de chasse, et qui, portés par leurs ailes, font jeu égal avec elles, et la rapide Iris, qui met fin à la poursuite, on ne sait qui va le plus vite, et qui sert de modèle aux autres. Pour le dire en termes rhétoriques, on voit bien que chacune de ces figures, situées sur un même axe paradigmatique, pourrait être le comparant des autres. Il en va de même pour les épopées postérieures, où les inévitables concours funéraires, avec leurs courses de char et leurs courses à pied, donnent lieu à des images désormais canoniques, faisant intervenir des chevaux fils de Borée, des sprinteurs aussi rapides que le zéphyr ou dépassant les cavales à la course, des biges laissant les vents derrière eux et volant si vite sur la plaine qu'ils n'impriment aucune trace sur le sol ${ }^{117}$.

On notera cependant qu'en reprenant ces thèmes homériques, les auteurs épiques des époques hellénistique et romaine les détachent de leur sens originel et, à quelques nuances près, ne connectent plus les images de la rapidité avec celle de l'excellence militaire. Au fond, le meilleur des Argonautes, ce n'est ni Iphiclos, ni Euphémos, ni l'un des Boréades, mais Jason.

Revenons donc aux textes homériques, où la vélocité se fait clairement la compagne de la vaillance. Il est alors frappant de voir tous ces thèmes converger vers ce qui constitue peut-être le clou de l'Iliade, le récit tant différé, tant attendu, de l'aristeia d'Achille et de son combat contre Hector. Dès le moment où le fils de Pélée revêt ses armes, "ce sont comme des ailes qui lui poussent alors et soulèvent le pasteur d'hommes ${ }^{118}$; dès lors les verbes de mouvement se multiplient, accompagnés d'un déchaînement de tous les éléments, vents en premier. Et quand on en vient à la rencontre avec le meilleur des Troyens, celui qui avait coutume de courir bien en avant des autres ( $\pi \rho 0 \theta \varepsilon ́ \varepsilon ı v)^{119}$, ce n'est pas un duel armé que décrit le poète, mais une course : Hector, pris de tremblements, n'a plus l'audace de rester ( $\mu \varepsilon ́ v \varepsilon ı v)$ à son poste et s'enfuit; Achille aussitôt le poursuit

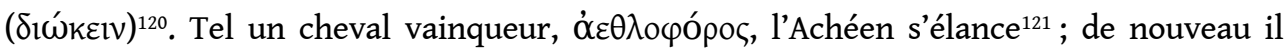

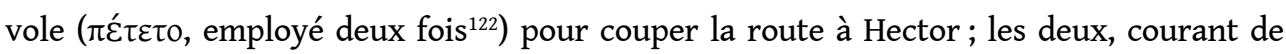
conserve (comme le montre l'emploi du duel $\pi \alpha \rho \alpha \delta \rho \alpha \mu \varepsilon ́ \tau \eta v)$ ), «ne luttent pas pour une

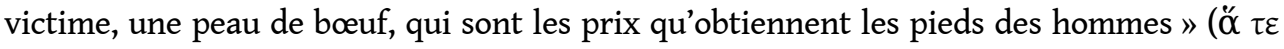

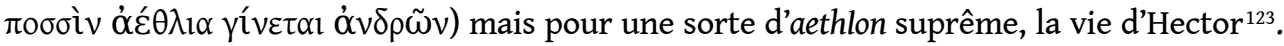
Et dès le vers suivant, une nouvelle comparaison agonistique prend le relais : les voici des chevaux aethlophoroi qui contournent la borne « car un grand prix les attend, un trépied ou une femme ». Ce concours de vitesse avait lieu devant des spectateurs de choix, l'assemblée des dieux ${ }^{124}$.

En choisissant la course comme clef de lecture du chant XXII de l'Iliade, on en vient donc à dégager la récurrence de thèmes attachés à la vitesse, avec ses connotations agonistiques, dans ce qui devait être le combat de force par excellence. Il est clair que cette course se

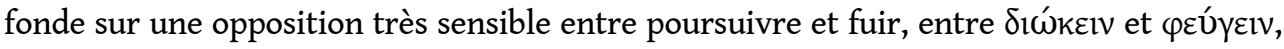
qui semble plus de mise dans le domaine de la cynégétique qu'au cœur de l'action hoplitique $^{125}$. De fait, lorsqu'on retrouve cette opposition à l'époque classique, c'est sous la plume d'un auteur tel que Xénophon, dans ses traités sur l'Art de la chasse, l'Art équestre, et sur le Commandant de cavalerie ${ }^{126 !} \mathrm{Au}_{\mathrm{IV}}^{\mathrm{e}}$ siècle, ces images de poursuite et de fuite semblent donc devoir être confinées au monde équestre ou à celui de la chasse, et si Xénophon entend redonner leurs lettres de noblesse à ces pratiques et valoriser leur apport guerrier, le fait est qu'il se plaint du peu d'intérêt qu'on leur accorde... 
Il peut alors être intéressant de remarquer que cette conjonction en Achille de la vitesse et de la valeur militaire est encore lisible sur quelques peintures de vases de la fin de l'époque archaïque et du début de l'époque classique. Plusieurs peintres se sont en effet attachés à traduire en images la prodigieuse rapidité du Péléide. Il se peut qu'une célèbre amphore provenant du groupe de Léagros le représente, sous la forme d'un guerrier ailé, volant au dessus de la mer et d'un navire - ou plutôt, courant, puisqu'il est figuré dans la position dite de la "course agenouillée » (Fig.1): nous retrouvons là quelques uns des thèmes explorés ${ }^{127}$. Mais la solution la plus simple et la plus fréquemment utilisée par les peintres consiste à juxtaposer sa course avec celle de chevaux, tout en signalant que la comparaison équestre tourne à l'avantage de l'humain; et c'est ce qu'on observe sur un motif qui connut un certain succès dans l'iconographie athénienne des $\mathrm{VI}^{\mathrm{e}}$ et $\mathrm{v}^{\mathrm{e}}$ siècles, le meurtre de Troilos par Achille. Peu évoqué par les sources littéraires, qui en font d'ailleurs un traitement un peu différent ${ }^{128}$, cet épisode figure sur plus d'une centaine de poteries athéniennes, à commencer par le fameux vase François, et se décline en trois types : l'embuscade d'Achille à la fontaine, la poursuite proprement dite, et le meurtre sauvage de Troilos près d'un autel ${ }^{129}$. Sur le second de ces types apparaissent régulièrement les mêmes éléments : au centre, deux chevaux côte à côte s'enfuyant au grand galop, l'un d'entre eux monté par un Troilos adolescent ; à côté, Achille, bondissant après lui, la lance ou l'épée brandie; et souvent, de l'autre côté, Polyxène courant vers Troie $^{130}$. Il ne fait donc aucun doute que le coureur rattrape le cavalier, d'autant que le peintre précise parfois son idée en représentant la jambe avant de l'Achéen au même niveau que la queue ou les jambes arrière des chevaux; et sur toute une série d'images Achille tient déjà d'une main les cheveux de Troilos, qui finira décapité (Fig.2). Sur quelques unes d'entre elles, la présence d'un pégase comme épisème d'Achille vient réactiver l'idée du vol ailé ${ }^{131}$.

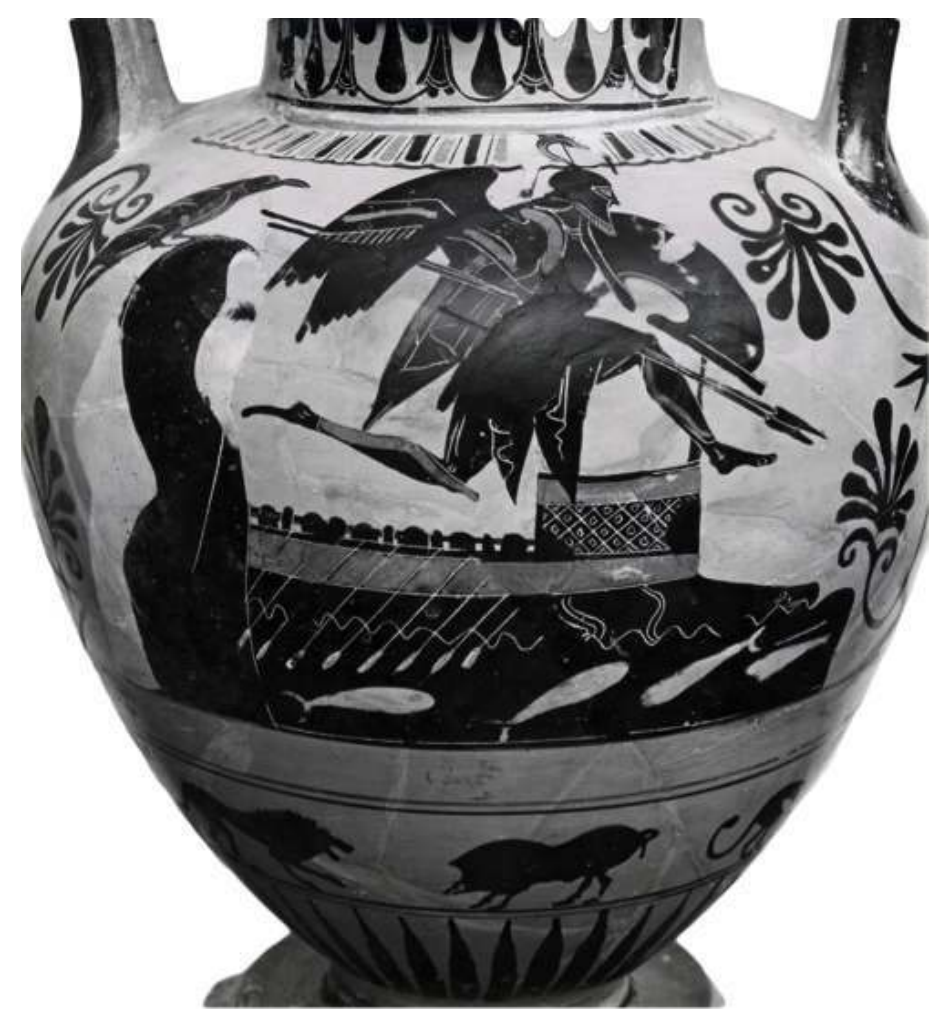




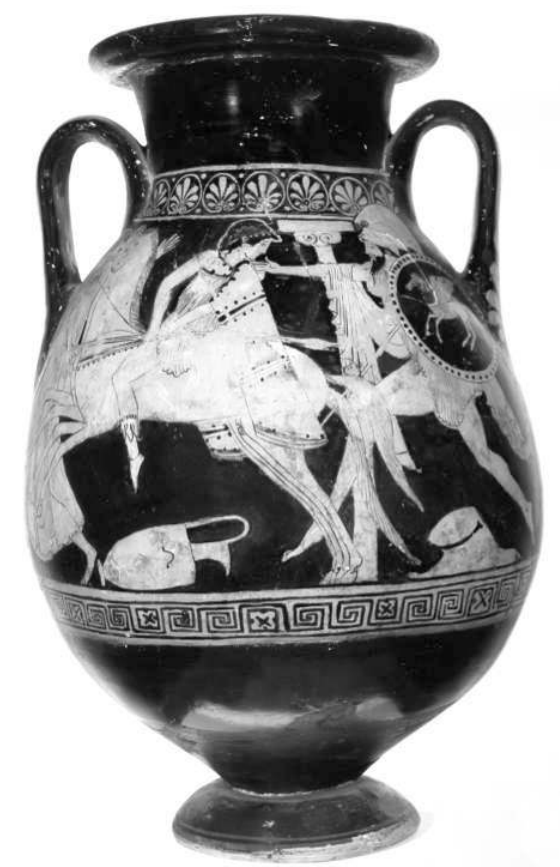

Figure 2

Achille poursuivant Troilos. Péliké attique à figures rouges, vers $475-450$ av. J.-C., Peintre du Louvre G231, Musée du Louvre, G231. ( ) Musée du Louvre, Dist. RMN-Grand Palais / les frères Chuzeville.

Certes, il s'agit d'une embuscade, d'un épisode rusé qui conduit à un combat déséquilibré. Là encore, nous sommes très près du domaine de la chasse - et la présence de chiens ou de lièvres dans le champ ne fait que renforcer cette connotation ${ }^{132}$. Quand on en vient à représenter le duel final de l'Iliade, la course disparaît la plupart du temps ${ }^{133}$, le duel se fait statique, et déjà Hector s'effondre blessé à mort. Toutefois, sur une série de vases provenant du groupe de Léagros ${ }^{134}$, la scène se décale légèrement sur le plan narratif, se focalisant désormais sur le cadavre d'Hector trainé par le char de son vainqueur. L'histoire est bien connue, mais l'iconographie y ajoute ses propres données, concentrant le maximum de sens en une seule image. On y voit ainsi l'eidôlon de Patrocle, et parfois sa tombe, une déesse ailée accourant à la rencontre de l'attelage, et surtout, un Achille non pas sur son char, mais, plusieurs fois, courant à ses côtés. Le héros bondissant est de nouveau représenté comme faisant jeu égal avec son attelage au galop, la jambe avant parfois confondue avec celles des chevaux, dans une impression de mouvement éventuellement redoublée par l'épisème de son bouclier, en forme de triskèle ou de fauve en mouvement (Fig. 3) ${ }^{135}$. Cette course d'Achille, complètement superflue sur le plan narratif, prend sens dès lors qu'on lui donne pour fonction de connoter la vélocité du héros : la victoire d'Achille, son triomphe sur Hector, s'expriment dans une manifestation de vitesse qui rappelle l'union indéfectible entre la valeur militaire de l'Achéen et la légèreté de ses pieds. 


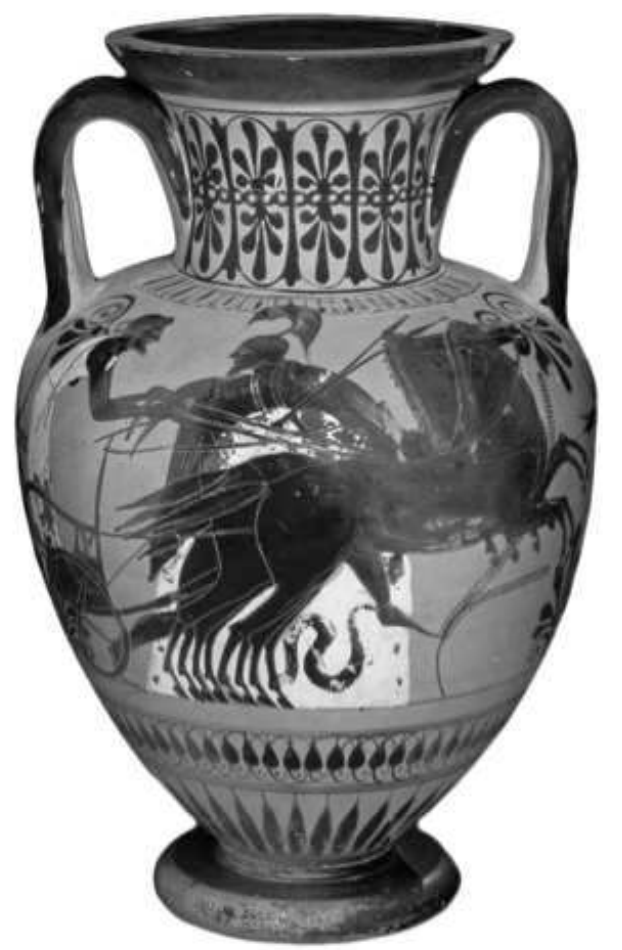

Figure 3

Achille courant entre la tombe de Patrocle et son char, auquel est attaché le cadavre d'Hector. Amphore attique à figures noires, vers 520-500 av. J.-C., groupe de Leagros, British Museum 1842,0314.2 (=B239). (c) Trustees of the British Museum.

\section{Voler à la victoire}

Ce que nous apprend donc le modèle achilléen, c'est qu'il existe une antique association entre puissance guerrière et vitesse, et qu'elle se manifeste par tout un réseau d'images, un jeu d'équivalence entre le héros rapide, le cheval, l'animal chasseur ou chassé, le vent, et toutes sortes de divinités ailées. Si ces images ont été incessamment reprises et retravaillées par les imitateurs d'Homère au sein du genre épique, on ne saurait les résumer, même aux époques tardives, à de simples topoi littéraires. Au contraire, elles semblent avoir imprégné en profondeur les mentalités, alors même que leur pertinence militaire semblait décroitre, et ne s'être nullement cantonné à l'épopée. Le monde de l'athlétisme, en particulier, semble les avoir parfaitement absorbées et développées.

Prenons ainsi l'idée du courir qui est un voler - certes peu originale en soi - dont nous avons vu la persistance dans la peinture de vase de la fin de l'archaïsme, voire dans l'œuvre de Xénophon. Près d'un millénaire après la composition de l'Iliade, l'érudit Philostrate, décrivant dans son traité sur le sport les techniques des différents types de coureurs, affirmait que ceux qui se spécialisaient dans le stade accéléraient leur course par le mouvement de leurs bras, "comme si c'étaient des ailes ${ }^{136}$. Revoici l'image de l'Achille volant ! Ce traité qui se veut scientifique porte en réalité les marques de la pensée mythique. 
De même, le Péloponnésien Ladas, olympionice au dolichos vers le milieu du ve siècle, fit l'objet d'une très célèbre statue de Myron, que vinrent commenter plusieurs épigrammes de date inconnue; d'après ces ekphraseis, l'athlète y semblait vivant, en équilibre sur la pointe des pieds, emporté par le vent ${ }^{137}$. Ce Ladas était, selon Pausanias, le plus rapide coureur de son temps, et son statut apparaît quasi-cultuel : soudainement malade après sa victoire olympique, il mourut sur le chemin du retour, près de Sparte, où il fut enterré ; sa disparition en pleine gloire lui valut une statue dans le temple argien d'Apollon Lycos, situé tout contre le dromos, ce qui n'est pas sans évoquer la position d'Achille à Élis ${ }^{138}$. Un second Ladas, un Achéen d'Aigion, vainqueur au stade à la cent-vingt-cinquième olympiade (280 av. J.-C.) et vedette incontestée des sprinteurs, fut également le laudandus d'une épigramme, qui proclamait ${ }^{139}$ :

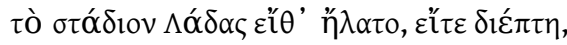

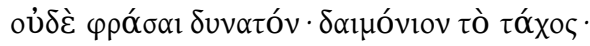

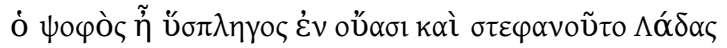

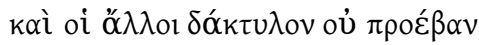

Ladas a-t-il couru ou survolé le stade?

Impossible à dire. Divine vitesse !

Le bruit de la barrière était encore dans les oreilles qu'on couronnait déjà Ladas

Et les autres concurrents n'avaient pas avancé d'un pouce.

51 Cette épigramme, qui fut suffisamment célèbre pour être parodiée par Lucilius ${ }^{140}$, n'est qu'un élément de la légende qui se développa autour de Ladas. Un autre élément transmis par Solin rapportait que le sprinteur, quand il courait, ne laissait aucune empreinte sur le sable de la piste; Catulle, pour sa part, l'englobait dans une liste qui comprenait des figures désormais bien connues, de Pégase aux Boréades, en passant par les cavales de Rhésos ou Persée aux talons ailés ${ }^{141}$. Ainsi se réunissent autour de ces deux Ladas, que la tradition semble avoir parfois confondus ${ }^{142}$, tous les thèmes homériques de la rapidité : le

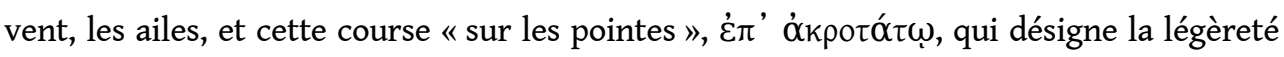
extrême du coureur, cette capacité à ne laisser quasiment aucune trace de son passage ${ }^{143}$. D'Achille à Ladas d'Aigion, ce sont donc les mêmes représentations qui s'attachent à la figure du coureur.

Cela dit, l'épigramme en l'honneur de Ladas nous permet peut-être de pousser un peu plus loin l'analyse de ces représentations. Les deux derniers vers créent une saisissante ellipse temporelle qui semble transformer ses concurrents en statue ${ }^{144}$; l'arrivée du coureur est contemporaine de son départ; il est ici et là dans le même temps. La distance et le temps semblent abolis par la course. L'anecdote de Solin ne dit pas autre chose : le sable n'a même pas le temps de retomber que Ladas est déjà passé. Si le sable, les flots, les épis des champs ne portent pas l'empreinte du coureur, c'est parce que la course nie radicalement l'espace auquel ils appartiennent, un espace du passage, pour ne retenir que les pôles de départ et d'arrivée.

Cette idée, on la retrouve explicitée par deux passages de Lucien, l'un sur le mode comique - et l'imitation de l'épigramme me paraît patente -, l'autre sur un mode plus sérieux et se voulant théorique. Commençons par la satire: dans le Timon, Ploutos 
explique à Hermès que sa rapidité est entièrement dépendante du type d'ordre qu'il a à exécuter. Si Zeus lui demande d'apporter la richesse, il est lent et traîne la patte. Mais s'il lui est demandé de l'ôter à quelqu'un, les ailes lui poussent, il est plus rapide que les songes ${ }^{145}$ :

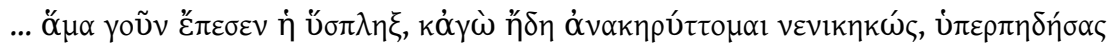

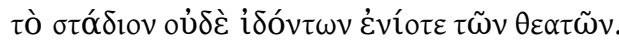

... la barrière n'est pas tombée que je suis déjà proclamé vainqueur, ayant franchi d'un bond tout le stade sans qu'aucun des spectateurs n'ait jamais le temps de rien voir.

Et voici maintenant la version théorique, qui rappelle Philostrate par sa volonté de rationaliser une image issue de la mythologie ${ }^{146}$ :

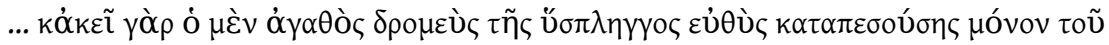

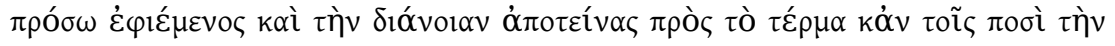

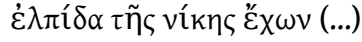

... car le bon coureur, à peine la barrière tombée, ne s'attache qu'à ce qui est devant lui, et tend tout son esprit vers la borne, confiant à ses pieds l'espoir de la victoire.

Dans ces deux textes apparaît l'image d'une victoire instantanée, succédant

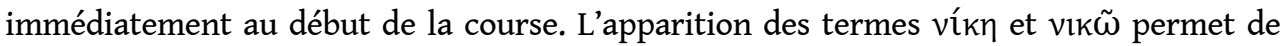
compléter ce que signifiait l'épigramme de Ladas. On a vu que son arrivée était contemporaine de son départ. Plus précisément, l'épigramme le montre couronné alors même que la barrière du départ résonnait encore du bruit de sa chute. L'abolition de la distance et du temps signifie une immédiateté de la victoire. Ce vol de Ladas vers son couronnement, il faudrait le rapprocher de celui de quelques divinités ailées, principalement Iris et Niké, toutes deux porteuses de victoire, et pour lesquelles tous les motifs de la course et du vol sont déployés; elles-mêmes ne sont ailées que pour permettre la réalisation immédiate, instantanée, de la volonté de Zeus ${ }^{147}$.

Peut-être sommes-nous en mesure de mieux comprendre, maintenant, comment l'imaginaire de la vitesse en fait une vertu particulièrement efficace dans le domaine guerrier. Courir vite, c'est d'une part exprimer sa biè; c'est, d'autre part, savoir se déplacer de façon instantanée. Il y a du surgissement dans l'arrivée - dans l'apparition, devrait-on dire - du coureur, un surgissement brusque et soudain qui se rapproche de

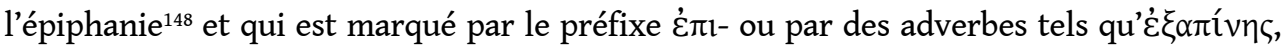

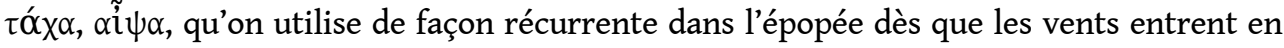
scène ${ }^{149}$, et dans la littérature militaire, pour décrire l'arrivée, souvent providentielle, de troupes fondant sur l'ennemi ${ }^{150}$. De l'épopée à l'histoire, le transfert se fait d'ailleurs aisément : au cap Artémision, en 480 av. J.-C., l'allié providentiel qui accourut au secours des Grecs n'était autre que le dieu Borée. La fantastique flotte perse fut soudainement

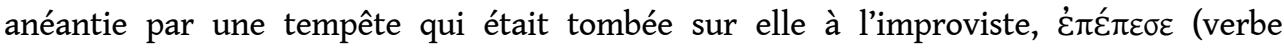
employé deux fois) ${ }^{151}$. Les Athéniens, qui le remercièrent en fondant un sanctuaire sur les bords de l'Ilissos, disaient que le dieu Borée leur était venu en aide, ßon $\theta$ ń $\sigma \alpha v \tau \alpha^{152}$. C'est

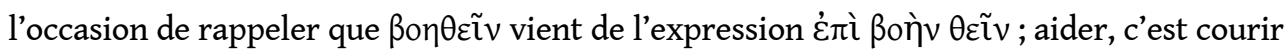
à l'appel du cri de guerre ${ }^{153}$. Il y a des chances pour que ce verbe $\theta \varepsilon \tilde{v} v$ ait résonné encore suffisamment fort dans le $\beta$ $\emptyset \theta \eta ́ \sigma \alpha v \tau \alpha$ des Athéniens remerciant le rapide dieu Borée. Ajoutons que le cap Sépias, où se trouvait stationnée la flotte perse, était consacré à la déesse Thétis ${ }^{154}$ : Achille n'est pas loin... 

mentionner son équivalent linguistique, ßon $\delta \rho \mu \varepsilon \tilde{v} v$, construit suivant le même principe. C'est de ce verbe que dérivent les noms du mois de boédromion, à Athènes, et de la fête des Boédromia. Or l'aition de la fête montre combien ce dromein était encore sensible dans le nom composé : il s'agirait en effet d'une commémoration de l'aide apportée en grande hâte par Ion (ou Xouthos, selon les sources) qui courut à l'aide des Athéniens et leur donna le salut ${ }^{155}$. Là encore, le surgissement rapide de forces alliées est venu apporter la victoire. Or c'est en ce mois de boédromion qu'une certaine tradition athénienne, au mépris de toute chronologie, situait la plupart des grandes victoires de l'histoire de la cité : la bataille de Marathon, au six du mois, celles de Mycale et de Platées le trois (jour des Nikétéria), le quinze la victoire navale de Naxos, et le vingt celle de Salamine ${ }^{156}$. Il est évident que ce regroupement dans un même mois ne repose sur rien d'historique, et que les victoires ont été attirées en boédromion par les connotations que comporte son nom.

Enfin, courir vite, c'est maîtriser le temps et l'espace qui nous sépare de la victoire. Or, cette idée de la victoire instantanée et de la distance abolie trouve une correspondance frappante dans une célèbre anecdote de la bataille du cap Mycale, dernier grand combat de la seconde guerre médique. Au moment où les soldats grecs partaient au combat, un caducée - symbole d'Iris ou d'Hermès - apparut sur la plage tandis qu'une rumeur volait

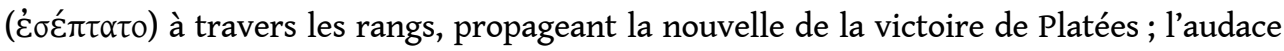
et l'ardeur des troupes en furent toute revigorées. Il apparut après enquête que les deux batailles avaient eu lieu le même jour; seul un prodige avait permis à la rumeur de franchir instantanément les centaines de kilomètres séparant les deux champs de bataille 157. Une semblable histoire était rapportée pour la victoire de l'Eurymédon: la rumeur l'avait annoncée à Athènes le jour même où elle eut lieu. Que ce soit après la bataille de Mycale ou après celle de l'Eurymédon que les Athéniens instaurèrent un culte pour Phémé, la rumeur, importe peu pour notre propos ${ }^{158}$. L'essentiel est de bien cerner cette structure où la victoire et son annonce se font simultanées, défiant la distance et le temps, et ce par l'intermédiaire d'un agent ailé.

À explorer ce type de récits, on ne peut que retomber vite sur des histoires où s'entremêlent guerriers et coureurs, batailles, épiphanies et concours olympiques. Prenons par exemple la bataille de la Sagra, qui opposa dans la première moitié du $\mathrm{VI}^{\mathrm{e}}$ siècle les Locriens épizéphyriens et les Crotoniates et qui s'accompagna d'une tradition riche en prodiges. En grande infériorité numérique, les Locriens avaient invoqué Ajax, le fils d'Oilée, et obtenu de Sparte l'envoi des Dioscures, des héros qui, de fait, étaient apparus sur le champ de bataille et avaient permis une victoire aussi spectaculaire qu'inattendue. Prodige supplémentaire, la nouvelle en avait été transmise le jour même

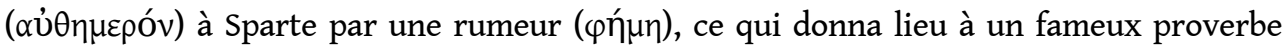
figurant dans tous les recueils parémiologiques. Or, Strabon nous livre une variante

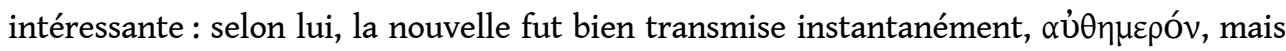
c'est à Olympie qu'elle parvint, non à Sparte, et le jour même du concours olympique. Après enquête, on s'aperçut que la nouvelle, dans sa rapidité, disait vrai ${ }^{159}$. Le rôle attribué aux Dioscures n'était peut-être étranger à ce décalage olympique ; après tout, Castor était censé avoir remporté l'épreuve de la course lors des proto-concours olympiques organisés par Héraclès ${ }^{160}$. Mais je crois surtout que c'est le propre des Olympia d'attirer ce genre de récits; pour Pline l'Ancien, c'est la destruction de Sybaris par Crotone qui fut annoncé le jour même à Olympie ${ }^{161}$. 
C'est qu'Olympie est le royaume de la course et des coureurs. Prenant le relais d'Iris ou de Phémé, le coureur, bien souvent, produit les mêmes effets que ces divinités. Par sa vitesse intrinsèque, il est le vecteur idéal de la victoire, qu'il vient annoncer et qu'il symbolise quasiment. Ainsi, l'Argien Aigeus, vainqueur olympique du dolichos en 328, aurait couru le même jour ( $\alpha \dot{\theta} \eta \eta \mu \varepsilon \rho o ́ v)$ jusqu'à Argos annoncer sa victoire, parcourant de la sorte une centaine de kilomètres en un temps record ${ }^{162}$. C'est en quelque sorte une version inverse, et pacifique, du proverbe de la Sagra : le coureur part d'olympie et annonce la victoire dans sa cité d'origine - mais l'idée est la même, associant dans un même mouvement la victoire, la course et l'instantanéité. Plus frappant encore, l'épreuve de l'hoplitodromie elle-même conserve dans son aition une idée semblable, et cette fois-ci dans un contexte militaire : selon Philostrate, ce concours trouvait son origine dans une guerre entre Élis et Dymé, guerre si féroce qu'elle ne respectait pas même la trêve olympique ${ }^{163}$ :

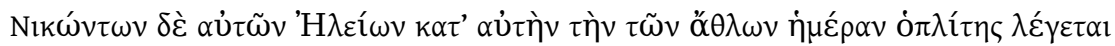

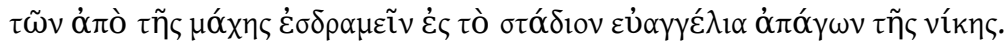

On raconte que les Éléens furent vainqueurs le jour même des épreuves, et qu'un hoplite courut de la bataille jusqu'au stade pour annoncer l'heureuse nouvelle de la victoire.

Enfin, comment ne pas évoquer un autre hoplitodrome, pas forcément plus historique que l'Éléen, qui s'était rendu célèbre pour une semblable annonce ?j'ai nommé le fameux coureur de Marathon, qui, le jour même de la victoire, courut en armes jusqu'à Athènes et

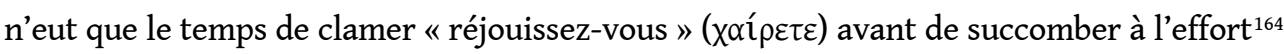
- Ce messager, nommé tantôt Philippidès, dans une évidente confusion avec l'hémérodrome qui fit l'aller-retour à Sparte avant la bataille, et tantôt Euklès, venait lui

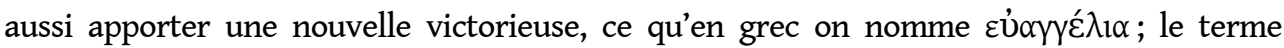
désigne également le rite qui lui est associé, un sacrifice d'action de grâce ${ }^{165}$. Pour célébrer celui de la victoire de Platées, l'oracle de Delphes ordonna d'éteindre le feu de tous les autels pollués par les Perses pour les rallumer ensuite depuis le foyer delphique. L'on eut recours là aussi à un coureur de longue distance : le Platéen Euchidas s'offrit à rapporter le feu de Delphes, courut l'aller-retour dans la journée et put allumer l'autel de Zeus Eleutherios, créé pour l'occasion, avant de s'effondrer mort ${ }^{166}$.

61 L'affinité des deux histoires est évidente: deux coureurs d'une vitesse surhumaine, annonçant la victoire ou permettant sa consécration rituelle, et, tel Ladas, mourant tous deux de leur exploit, en pleine gloire. Il est intéressant de voir mêlée à cet ensemble thématique l'antique signification de la course olympique, qui donnait pour fin à l'épreuve du stade d'allumer l'autel de Zeus. Bref, il ne paraît guère probable que cet Euchidas, dont Plutarque nous fournit la première et unique mention, ait eu une quelconque historicité ; au vrai, toute l'histoire apparaît comme l'aition d'une autre course armée, celle des Eleutheria, qui redéploie de manière remarquable tous les thèmes ici abordés. Fête panhellénique commémorant la bataille de Platées, probablement fondée à l'époque d'Alexandre et de la ligue de Corinthe, les Eleutheria comprenaient les épreuves gymniques et hippiques habituelles ${ }^{167}$, mais leur point d'orgue était sans conteste la longue course armée qui partait du trophée de la bataille pour aboutir, semble-t-il, à l'autel de Zeus Eleutherios, ce qui représentait une distance d'une quinzaine de stades. À ce symbolisme qui joint la victoire sportive à l'euangelia s'en ajoute un autre, par lequel Achille réapparaît : le vainqueur de l'épreuve recevait le beau titre d'aristos Hellènôn et se voyait attribuer l'aristeion; et comme il était inconcevable que le meilleur des Grecs pût 
être vaincu, le règlement spécifiait que tout vainqueur qui se représentait à l'épreuve devait vaincre ou mourir ${ }^{168} . .$.

Vaincre ou mourir, voire vaincre et mourir, moyen idéal d'obtenir un kléos impérissable ${ }^{169}$ , ces slogans s'adaptent aussi bien au monde guerrier qu'à l'esprit de l'agonistique sportive. Aussi n'est-ce pas un hasard si la tradition la plus répandue donnait le nom d'Euklès au coureur de Marathon, bataille au terme de laquelle les Athéniens consacrèrent un sanctuaire à la déesse Eukleia, ni si Euchidas, à sa mort, fut enterré dans le sanctuaire platéen d'Artémis Eukleia ${ }^{170}$.

\section{Course et guerre hoplitique : conclusions}

Il suffit donc de lancer quelques lignes dans l'océan historiographique des guerres grecques pour en retirer une série d'anecdotes plus ou moins légendaires, attachées à une bataille donnée, qui associent, dans ce contexte militaire, la course et la victoire, au point que la première semble précipiter la seconde. Ces anecdotes, fondées sur le thème de la rapidité qui annule la distance et le temps, obéissent à une structure qu'on retrouve également dans le monde agonistique; elles se traduisent à l'occasion par des courses armées, avec, à l'arrière-plan, l'idée que l'hoplite vainqueur reproduit la figure d'Achille, le meilleur des Achéens.

Pour autant, dans le monde des citoyens-hoplites, il était plus difficilement concevable de faire de la vitesse le mode fondamental du combat ; la phalange, limitant les mouvements et exaltant le fait de tenir bon plutôt que celui de courir, ne laissait guère de place à un Achille ${ }^{171}$. Dans ce contexte, la course armée était sans doute moins un exercice militaire pertinent qu'une manière de résoudre, avec un certain décalage, la contradiction qu'il existait entre un héritage épique et une réalité hoplitique substantiellement différente ; la figure de l'hoplite coureur venant réconcilier l'une et l'autre ${ }^{172}$. Quant à l'historiographie antique, hésitant elle aussi entre idéologie homérique et volonté de réalisme, elle tente différents moyens de concilier les deux. Aussi assiste-t-on à tout un jeu de transpositions et de décalages qui rejettent la course à la périphérie de la bataille, c'est à dire à son commencement, avec la charge, et à sa fin, avec la poursuite et l'annonce de la victoire, sans pour autant que cette course perde les valeurs qui lui sont traditionnellement attribuées. Exemplaire, à cet égard, est le récit que donne Hérodote de la bataille de Marathon: alors que le cœur du combat est expédié en quelques lignes, toute la gloire est concentrée sur la charge héroïque des Athéniens, qui auraient couru les huit stades qui les séparaient des Perses tout en gardant leur formation (exagération manifeste) ${ }^{173}$, et sur le retour précipité vers Athènes, qui coupa court à la tentative de débarquement de Datis. Et Hérodote de décrire ainsi cette dernière action

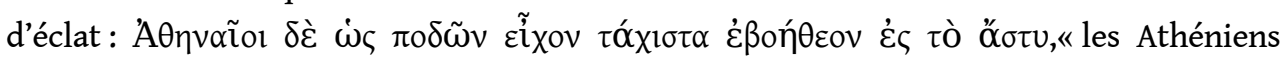
coururent de toute la vitesse de leurs jambes au secours de la ville ${ }^{174}$. J'avais également rappelé, au début de cette recherche, la charge des Thébains lors de la bataille de Leuctres ; c'est maintenant sans surprise qu'on apprend que, selon Plutarque, le bataillon

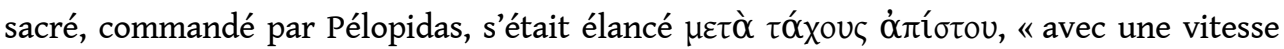
incroyable ». La victoire ne pouvait que suivre ${ }^{175}$.

Et puis, à côté de ces armées de soldats coureurs, on rencontre encore quelques Achilles individuels: des chefs de guerre dont les exploits sont inséparables de leur grande vélocité. Je pourrais mentionner Alexandre, imitateur conscient du Péléide tout au long 


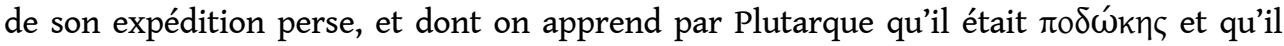
aurait pu concourir sur le stade olympique; ce qu'il refusa avec dédain, souhaitant n'avoir que des rois comme adversaires ${ }^{176}$. Mais l'imitation d'Achille par Alexandre est trop volontaire, trop manifeste, et je préfèrerai conclure sur une autre figure, moins ouvertement mythifiée et puisée dans l'univers rationaliste d'un Thucydide, celle du Spartiate Brasidas. Dans cette période de changements, notamment militaires, que constitue la guerre du Péloponnèse, et dans cette œuvre qui se veut hostile au mythodès, il me paraît possible de déceler la persistance des représentations épiques. Comme l'a remarqué Simon Hornblower, dès son apparition dans l'œuvre de Thucydide, le général est caractérisée par une série de verbes de mouvements et de termes liés à la vitesse - chose assez originale si l'on se rappelle la lenteur proverbiale de la phalange spartiate ${ }^{177}$. Or, avant de décéder à Amphipolis et d'y recevoir les honneurs cultuels d'oiciste et de sauveur de la cité, Brasidas fut accueilli en fête par les habitants de Scioné qui « s'avancèrent à sa rencontre comme on fait pour un athlète victorieux " ${ }^{178}$. Athlète et coureur, Brasidas serait-il un hoplite aux pieds légers? L'Alcibiade du Banquet nous fournit la réponse ${ }^{179}$ : «Des semblables à Achille, il y en eut : on pourrait lui comparer, entre autres, Brasidas ».

\section{NOTES}

* Cet article a bénéficié des conseils de François de Polignac, de Pierre Ellinger et de John Ma, que je tiens à remercier chaleureusement ; je reste évidemment seul responsable des propos que j'y tiens.

1. CIO, Charte Olympique, éd. du 8 juil. 2011, p. 10. La Fondation Internationale pour une Trêve Olympique (FITO) est une ONG crée par le CIO en juillet 2000. ONU : voir particulièrement les résolutions $48 / 11$ du 25 oct. 1993 ; 64/4 du 19 oct. 2009 ; et 66/5 du 11 oct. 2011.

2. Voir par exemple GOLDEN (2008), p. 136-142, et ROUGEMONT (1973) pour une étude rigoureuse des différentes trêves festives connues.

3. Riche étude de CROWTHER (2004), p. 313-321. Voir également SANI (1982), p. 53-57; ROBERT (1989), p. 334-335 ; PERYSINAKIS (1990), p. 43-49; MÜLLER (1995), p. 58-68 ; ID. (1996), p. 43-47 ; MANN (1998), p. 7-21; SCANLON (2002), p. 13-15.

4. Ainsi Golden (1998), p. 23-28 et MANN (1998), p. 11-13. Mais voir cependant EvJEN (1992), p. 103-104, et surtout POLIAKOFF (1987), p. 94-99, dont certaines remarques sur la place accordée aux athlètes dans l'armée ne doivent pas être négligées.

5. Voir FINLEY - PLEKET (2008), p. 185-187 ; POLIAKOFF (1987), p. 99-103 ; ANGELI BERNARDINI (1980), p. 83-88.

6. Euripide, fr. 282 (TGrF, KANNICHT).

7. Lucien, Anacharsis, 24 ; Dion Chrysostome, Discours 29 ; Voir également Diodore, XV, 50, 5, et Plutarque, Propos de table II, 5, 2 (Mor., 639e). Cf. FINLEY - PLEKET (2008), p. 175-176 et 193-194; POLIAKOFF (1987), p. 94-99.

8. Voir par exemple Hérodote, V, 47 (Philippos) ; V, 102 (Eualcidès) ; VI, 92 (Eurybatès) ; VIII, 47 (Phayllos); IX, 105 (Hermolycos); Xénophon, Helléniques II, 4, 33 (Lacratès); VII, 1, 33 
(Antiochos) ; Diod., XVI, 44, 3 (Milon) ; Pausanias, IV, 17, 9 (Phanas) ; VI, 8,6 (Timasithéos) ; X, 34, 5 (Mnasiboulos), etc.

9. Les deux faits sont réunis par Plut., Propos de table II, 5, 2 (Mor., 639e). Autres sources dans KURKE (1993), p. 133-137, qui, après VERSNEL (1970), p. 155-163, y voit des attestations d'une espèce de mana attaché à l'athlète victorieux, désigné par le terme de kudos et symbolisé par la couronne.

10. Platon, Lois VIII, 832e-833c.

11. Diod., XV, 30, 1-2 et 50, 5 ; Plut., Pélopidas, 4,1 et 7, 5. Cf. Théophraste, Histoire des plantes X, 20, 5 et Plut., Démon de Socrate, 25 (Mor., 594c).

12. Nepos, Épitomé, 2, 4: postquam ephebus est factus et palaestrae dare operam coepit, non tam magnitudini uirium seruiuit quam uelocitati : illam enim ad athletarum usum, hanc ad belli existimabat utilitatem pertinere. Cf. 5,4 .

13. Il est vrai que, selon Plutarque, Apophtegmes, Épaminondas 4-6 (Mor., 192d), Épaminondas condamnait la sarcophagie, c'est-à-dire le régime typique des athlètes lourds ; mais peut-être faut-il y voir avant tout l'expression de ses convictions pythagoriciennes ; cf. sur ce point VIDALNAQUET (1991), p. 112-113. Sur la condamnation des athlètes lourds dans la littérature savante, voir POLIAKOFF (1987), p. 93 ; KÖNIG (2005), p. 254-300 ; BRULÉ (2006), p. 276-279.

14. IG VII, $2462=$ CEG $632=$ RHODES - OSBORNE (2003), no 30, v. 9.

15. Voir le commentaire de TUPLIN (1987), p. 103-107, ainsi que celui de RHODES - OSBORNE, ibid.

16. Sur l'hoplitodromie, voir par exemple MILLER (2004), p. 32-33.

17. Plat., Lois VIII, 832e.

18. Philostrate, Gymmastique, 7 . Voir infra, $\S 5$.

19. MORETTI (1953), p. 21-23 ; BILIŃSKI (1960), p. 23-25. Ce qui est sûr, c'est que, d'après le témoignage des amphores panathénaïques, le concours était pratiqué à Athènes au moins aussi tôt qu'à Olympie : voir KYLE (1987), p. 181-182.

20. Tyrtée, fr. 11 et 12 (éd. PRATO) : voir particulièrement la déconsidération du coureur fr. 12, v. 2 et $4:$ il existe bien une "mauvaise course ", aux yeux de certains théoriciens de l'hoplitisme. Près de trois siècles plus tard, Platon ne dit pas autre chose : Lachès, 190e. Cf. DETIENNE (1999), p. 169-174; LAZENBY (1991), p. 91-97.

21. DONLAN - THOMPSON (1976), p. 339.

22. Sur cette bataille, voir principalement LAZENBY (1985), p. 151-162, et TUPLIN (1987), p. 72-107.

23. Diod., XV, 55, 3. Il s'agit sans doute de la même charge que celle du bataillon sacrée, mentionnée par Plutarque, Pélopidas, 23, 3.

24. Cf. Xén., Hell. VI, 4, 13.

25. Xén., Hell. VI, 4, 13-14 ; Diod., XV, 55, 4-5 ; Paus., IX, 13, 10.

26. Le pas supplémentaire : Polyen, II, 3, 2, avec BUCKLER (1985), p. 140-143. Sur ce cœur de la bataille où les deux fronts luttent bouclier contre bouclier, voir LAZENBY (1991), p. 87-109.

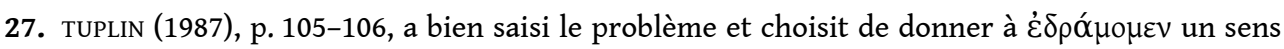
général désignant l'action militaire, sans toutefois en étudier les implications.

28. Voir les études de DONLAN - THOMPSON (1976) ; GARLAND (1992), p. 47-54; ELLINGER (2002).

29. Huit stades : Hdt., VI, 112. Réfutation par DONLAN - THOMPSON (1976). Cette valorisation de la charge par Hérodote semble donc très exagérée, d'autant qu'elle ne fut peut-être même pas la première : cf. WEES (2004), p. 172 et 180.

30. Hdt., VI, 113.

31. Plut., Propos de table II, 5, 2 (Mor., 639e). Cf. aussi Luc., Anacharsis, 24.

32. Xénophane, 21 B 2, 17-19 DK.

33. Sur l'évolution du programme olympique et cette primauté de la course, voir LEE (1988), p. $110-115$.

34. IG II $^{2} 2311$, avec les restitutions de SHEAR (2003). 
35. Sur la liste des prix des concours d'Aphrodisias (CIG II, 2758), datée du II ${ }^{\mathrm{e}}$ siècle ap. J.-C., le pancrace est, de loin, l'épreuve la mieux rémunérée; l'on notera également que les xystarchoi d'époque romaine étaient fréquemment d'anciens pancratiastes : cf. SCANLON (2002) p. 56 ; GOLDEN (2008), p. 34-35.

36. Inscriptions honorant de grands coureurs d'époque hellénistique et romaine : par exemple MORETTI (1953), no $45 ; 59 ; 66 ; 86$; et no 76 , une très grandiloquente inscription à la gloire de M. Aurelius Abas. Sur le développement des Eleutheria à époque tardive, voir en dernier lieu VAN NIFJ (2005) ; sur les Capitolia : CALDELLI (1993) ; RIEGER, (1999), p. 171-203. Voir également MORETTI (1987), qui montre la vigueur de l'école de sprint alexandrine à l'époque impériale.

37. Les critiques sont venues tôt: GARDINER (1910), p. 56. Plus récemment, Mark Golden s'est montré lui aussi sceptique et a supposé que la rivalité entre épreuves hippiques et gymniques a conduit à l'oblitération des premières par Hippias d'Élis : GOLDEN (1998), p. 41-45; scepticisme plus général (mais concernant davantage la datation que l'évolution du programme) chez CHRISTESEN (2007). LEE (1988) ; MILLER (2004), p. 226-227 ; KYLE (2007), p. 107, tiennent une position plus fidéiste.

38. "If any athletic event does have relevance to divine worship, that event is running " : LEE (1988), p. 113 ; voir aussi BURKERT (2005), p. 128

39. Voir notamment JÜTHNER (1968), p. 134-156, et BURKERT (1985), p. 61-62.

40. Philstr, Gym., 5-6.

41. BURKERT (2005), p. 127-130.

42. Ibid., p. 130-135, et p. 118-126 pour les histoires du mont Lycée, dont l'interprétation forme la matrice de tout le chapitre. Depuis, les loups-garous arcadiens se sont vus appliquer plusieurs types de grilles de lecture (voir la synthèse de JosT [2005]) et le rapprochement avec le sacrifice du bélier à Pélops peut ne pas paraitre évident.

43. JeAnMAire (1939), p.413-418; cette théorie a été brièvement reprise par BRELICH (1969), p. 449-456, qui lui a donné sa forme canonique. La référence à l'initiation est implicite dans BURKERT (2005), p. 134, et explicite dans ID. (1985), p. 106.

44. BRELICH (1969), ibid.; bURKERT (1985), ibid. De fait, une des interprétations antiques de l'épreuve de l'hoplitodromie, qui concluait les concours olympiques, en faisait un symbole de la fin de la trêve olympique et du retour à la vie ordinaire : Philstr., Gym., 7.

45. Mallwitz (1988), p. 86-89, pour le Pélopion, et p. 94-102 sur le stade. Pour situer le stade primitif, l'archéologue se fonde sur la position des puits du viI ${ }^{\mathrm{e}}$ siècle jouxtant la piste. Il faut toutefois noter que ces puits ne disent rien de l'Olympie du viII ${ }^{\mathrm{e}}$ siècle (raison pour laquelle A. Mallwitz fait débuter les concours vers 700). SCANLON (2002), p. 32-39, fait ainsi l'hypothèse que le tout premier stade n'était nullement une construction spécifique mais une simple piste tracée au sol. Ce savant insiste également sur le rattachement symbolique à l'Altis que constitue le tunnel d'entrée des coureurs, véritable cordon ombilical entre le stade et le sanctuaire !

46. Voir les mises en garde de CALAME (1992) et les importantes études réunies par FARAONE DODD (2003) - particulièrement celle de Fritz Graf, p. 3-22.

47. Dromos à Sparte et à Argos : MARCHETTI (1996), p. 155-165; à Athènes : TRAVLos (1988), p. 422428 ; KYLE (1987), p. 57-64; à Corinthe: WILLIAMS - RUSSEL (1981), p. 2-15; en Macédoine: HATZOPOULOS (1994), p. 55-62.

48. Paus., III, 14,6 .

49. Les courses de jeunes filles constituent un sujet à part entière, qu'on ne saurait traiter ici. Je renvoie notamment à CALAME (1977), passim ; ANGELI BERNARDINI (1988), p. 153-185 ; SCANLON (1984), p. 77-90 et ID. (2002), p. 98-199. Disons simplement qu'elles rejoignent certaines courses initiatiques spartiates et crétoises en ce qu'elles semblent bien être un rite prénuptial. Il n'est pas sûr, cependant, que l'aspect compétitif y soit aussi développé, et on notera que, dans les mythes 
qui leur servent d'aition, les coureuses doivent être vaincues pour être épousées, comme si la victoire à la course devait in fine rester le fait d'un homme...

50. Le programme gymnique comprenait stade, diaulos, dolichos, mais aussi makros (une course d'endurance) et pente dolichos ! Voir MILLER (2004), p. 146.

51. Plat., Lois I, 625d.

52. Le texte de base est Ephore FGrHist 70 F 149 (éd. JACOBY), apud Strabon X, 4, 21 (C482-484). Voir particulièrement les études de BRELICH (1969), p. 199 ; 204-205 ; VIDAL-NAQUET (1991), p. 167 ; TZIFOPOUlos (1998), p.137-171; WALDNER (2000), p. 222-242. Sur le verbe $\dot{\varepsilon} \gamma \delta \rho \alpha \mu \varepsilon \tilde{v} v$ et le vocabulaire des classes d'âge crétoises : BILE (1992), p. 11-18; CHANIOTIS (1996), p. 19-21 et 124.

53. Ce sont en tout cas les seuls exemples où l'interprétation initiatique trouve grâce aux yeux des auteurs de FARAONE - DODD (2003).

54. L'homme fait - ou, peut-être, le jeune adulte. Il se peut en effet que ce terme de dromeus ait désigné des Crétois ayant quitté le stade éphébique mais n'étant pas encore entrés dans leurs pleins droits de citoyens ; cf. CALAME (1977), p. 350-352 ; PETTERSSON (1992), p. 66-72; TZIFOPOULOS (1998), p. 150-169. Je remercie également John Ma d'avoir attiré mon attention sur les ekdromoi spartiates, terme apparemment technique qui désigne des hoplites adultes, mais issus des classes d'âges les plus jeunes, envoyés combattre hors des rangs - notamment contre les peltastes - en raison de leur mobilité : voir par exemple Thucydide, IV, 125, 3, ou Xénophon, Helléniques IV, 5, 16.

55. Voir hatzopoulos (1994), p. 87-89.

56. VIDAL-NAQUET (1991), p. 167 : « la course, en effet, est virile par excellence ».

57. Sur cette coutume de la statue olympique, voir RASCHKE (1988), p. 38-42 ; HERRMANN (1988), p. 119-137.

58. Mageiros : Athénée, IX, 28, 382b. Sa tombe : Paus., V, 8, 6 et VIII, 26, 3.

59. Young (1984) avait voulu voir en lui une preuve que les athlètes provenaient des classes populaires aussi bien qu'aristocratiques. Mais cette opinion a été réfutée plusieurs fois par H.W. Pleket (voir par exemple PLEKET [2001], p. 166-167).

60. Voir POLIGNAC (1979), p. 12-13, et ID. (1996), p. 172.

61. Paus. I, 43,7 .

62. Déjà par BELOCH (1912), I, 2, p. 149 ; voir aussi MORETTI (1957), no 1, p. 59 ; FONTENROSE (1968), p. 82-83.

63. MORETTI (1957) no 16, p. 61-62.

64. Paus., I, 44, 1. L'épigramme, attribué à Simonide, n'est conservée que partiellement par la tradition littéraire (scholie àThucydide I, 6, 5 = A. Plan. 272). Quant à l'inscription (IG VII, 52), elle fut manifestement regravée à l'époque d'Hadrien. Sur le problème des datations discordantes d'Orrhippos, voir FIGUEIRA (1985), p. 272-273. La question si débattue de l'invention de la nudité ne nous concerne pas directement ici : je me contente de renvoyer à GOLDEN (1998), p. 65-69 et SCANLON (2002), p. 199-273 avec la bibliographie qui y est mentionnée, et noterai simplement que cette pratique semble avoir été associée avec le domaine militaire et l'entraînement des soldats (voir notamment CHRISTESEN [2002], p. 30-31).

65. Sur les cinq vainqueurs précédant Oibotas, voir MORETTI (1957), no 1-5, p. 59-60.

66. Paus., VII, 17, 13-14.

67. Paus., VII, 17, 13. Le texte de Pausanias étant malheureusement corrompu à cet endroit, la nature du monument dépend des restitutions qu'on adoptera. Le terme $\mu v \tilde{\eta} \mu \alpha$, proposé par Michel Casevitz (CUF, 2000), me paraît à la fois conforme aux habitudes du périégète et plus

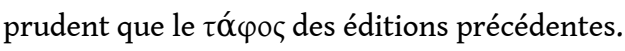

68. Sur Phanas et la valeur de ce type d'exploit sportif, voir Young (1996), p. 188-193.

69. Icaros : MORETTI (1957) no 28, p. 63 ; Phanas : no 142, p. 77 ; Pataicos : no 171, p. 81. 
70. Avec quelques précautions, cependant, car les deux termini ne se situent pas à au même niveau : la légende de la malédiction d'Oibotas a nécessairement été inventée après la victoire de Pataicos, mais pas obligatoirement avant celle de Sostratos ; cette dernière ne fait office de borne chronologique qu'à l'intérieur du récit légendaire. Il est cependant vraisemblable que le culte d'Oibotas et la tradition de couronner sa statue aient été instaurés au moins dès l'époque de Sostratos.

71. Paus., VI, $3,8$.

72. Hdt., VIII, 73 (les cités péloponnésiennes neutres); IX, 28 (dénombrement des troupes grecques à Platées) ; Paus., VII, 6, 4 (absence des Achéens aux grandes guerres panhelléniques).

73. Voir par exemple la recension d'HARRISON (2000), p. 82-92.

74. Cf. PolignaC (1979), p. 8, et Figueira (1995), p. 271-273 pour la datation et le contexte.

75. Ce sont Philippos de Crotone, Cléomédès d'Astypaleia, Euthymos de Locres, Théogénès de Thasos, Eutyclès de Locres, Diognétos de Crète et Pouludamas de Scotoussa, auxquels on a parfois ajouté Hipposthénès de Sparte, Glaucos de Carystos, Taurosthénès d'Égine, voire Attalos de Pergame. Voir les différentes propositions de listes de POLIGNAC (1979), p. 5-8 et 10; BENTZ - MANN (2001), p. 230-232 et CURRIE (2005), p. 120-157.

76. Schéma proposé par FONTENROSE (1968), p. 76-79; voir aussi BENTZ - MANN (2001), p. 232-233.

77. Sur le rôle essentiel joué par la statue dans le culte de l'athlète, voir les études citées note précédente de FONTENROSE et de BENTZ - MANN, ainsi que les articles à paraitre d'AzOULAY et de POLIGNAC.

78. L'étude pionnière est celle de POLIGNAC (1979) ; depuis, voir principalement HODKINSON (1999) ; KURKE (1993), p. 143-155 et surtout 153-155 ; MANN (2001), p. 34-36 ; BENTZ - MANN (2001), p. 230240 ; CURRIE (2005), p. 124-126.

79. KURKE (1991). Pour une analyse très éclairante du sens de kudos, cette aura de victoire donnée par les dieux, voir JAILLARD (2007).

80. Pour reprendre le titre d'un article fondamental de Leslie Kurke : KURKE (1993).

81. C'est la thèse principale de POLIGNAC (1979).

82. Comme le propose figueira (1985), p. 272-273.

83. Voir supra, n. 71.

84. Voir CUCHE (2011), p. 104-106.

85. Hdt., VIII, 47 ; Paus., X, 9, 2. Son épigramme dédicatoire : IG I $\mathrm{I}^{3} 823=$ MORETTI (1953), no $11=$ CEG no 265. De fait, Hérodote semble gratifier Crotone du mérite de son ressortissant ; bien plus tard, Alexandre, après la victoire de Gaugamèles, ordonna qu'on rebâtît Platées, cité symbole de la lutte contre les Perses, et qu'on envoyât une partie du butin à Crotone, en mémoire de l'aretè de Phayllos (Plut., Alexandre, 34, 2-3).

86. Tout au plus peut-on noter que, selon Pausanias, VII, 6, 4, les Achéens auraient souhaité participer à la bataille, mais seraient arrivés en retard. La vitesse d'Oibotas l'assurait en tout cas d'une arrivée en temps et en heure!

87. Comme le notent avec raison BENTZ - MANN (2001), p. 236, et surtout CURRIE (2005), p. 126-129.

88. À l'exception, peut-être, du cas très particulier des tyrans siciliens, Gélon et Hiéron ; mais il faut sans doute interpréter leur culte comme étant celui d'un oiciste plutôt que d'un athlète. Que l'on ne dispose d'aucun culte fermement attesté pour les propriétaires de quadriges vainqueurs est à mon sens une sérieuse difficulté de la thèse principale de CURRIE (2005) dans la mesure où la plupart des commanditaires des épinicies appartiennent à cette catégorie.

89. Pugilat: Euthymos, Théogénès, Diognétos, Cléomédès. Pancrace : Poulydamas, Théogénès. Euthyclès était un pentathlète. La spécialité de Philippos de Crotone est inconnue. Parmi les cas plus douteux, Glaucos et Attalos sont des boxeurs ou pancratiastes; Hipposthénès et Taurosthenès sont des lutteurs. 


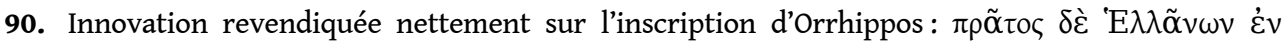

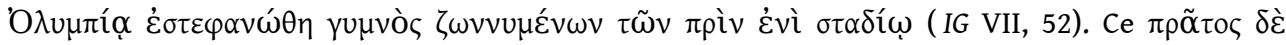
'E $\lambda \lambda \tilde{\alpha} v \omega v$ rappelle une formule devenue courante pour signaler ce que youNG (1996) considère comme l'équivalent antique des records sportifs.

91. Sur le skamma, ou aire de combat, les juges pouvaient tendre des barrières pour forcer les boxeurs qui évitaient trop le combat à se rapprocher l'un de l'autre : cf. POLIAKOFF (1987), p. 79-80. 92. POLIAKOFF (1987), p. 85-88 ; 90-93 ; FINLEY - PLEKET (2008), p. 66-70.

93. Sur la Biè Héracleiè : NAGY (1994), p. 366-367, avec, en arrière-plan, DUMÉZIL (1985), p. 71-131. Héraclès victorieux du pancrace : [Orphée], Argonautiques, 583-584 ; Paus., V, 8, 4 ; Hyg., Fab., 273, 10. En 212 av. J.-C., Capros d'Élide, vainqueur de la lutte et du pancrace aux mêmes concours olympiques, se vantait d'être "le premier depuis Héraclès " à obtenir cette double couronne. L'expression s'institutionnalisa : youNG (1996), p. 184-185.

94. FONTENROSE (1968), p. 85-87 (l'article entier serait à citer); MERKELBACH (1970), p. 47-49 ; DOUGHERTY (1993), p. 193-194; CURRIE (2002), p. 35-41 ; ID. (2005), p. 132-136.

95. Voir notamment BILINSKI 1960, p. 3-4.

96. Paus., VI, 11, 5. L'épisode est crédible : sur la base delphique de Théogénès, qui ne mentionne évidemment pas chacun des 1300 succès du Thasien, figure une autre victoire au dolichos, aux Hécatombaia d'Argos : MORETTI (1953), no 22. Luigi Moretti propose une autre explication, qui n'est pas nécessairement en contradiction avec celle de Pausanias : vaincu par forfait au pancrace par un nommé Dromeus de Mantinée, que Moretti propose d'identifier avec Dromeus de Stymphale, double périodonice du dolichos, Théogénès aurait souhaité prendre sa revanche sur le terrain de son rival. Même si l'identification des deux Dromeus n'a rien d'assuré, le nom même du Mantinéen aurait pu motiver Théogénès.

97. Achille et le dromos d'Élis : Paus., VI, 23, 2-3 avec JACQUEMIN (2002), p. 286-287. Il existait d'ailleurs un lieu nommé le dromos d'Achille, en Tauride, assez réputé des voyageurs : Hdt., IV, 76 et Denys, Périégèse, 306-307, pour les témoignages antiques; TUNKINA (2007), p. 225-233, pour sa probable localisation sur la presqu'île de Tendra. L'Achilleion lié aux combats rituels du Platanistas se trouvait hors de Sparte, sur la route de l'Arcadie : cf. Paus., III, 20, 8 avec MUSTI TORELLI (1991), p. 258-259.

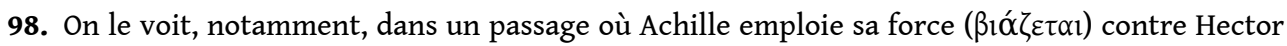
en le poursuivant de ses pieds rapides ( p. 374-375.

99. Homère, Odyssée XI, 290. Son fils Podarkès : Hésiode, Catalogue, fr. 199 (éd. MERKELBACH-WEST), v. 5 et schol. Od. XI, 326. Suivant la coutume, le nom du fils renvoie à une qualité du père. Sur le

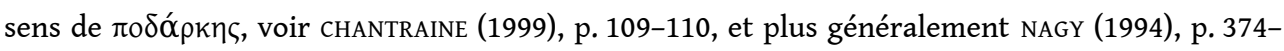
375.

100. Hom., Iliade VII, 138-141. Areithoos était, selon Phérécyde, FGrHist 3 F 158, le meilleur (aristos) des hommes de l'époque, ce qui le rapproche d'Achille, le meilleur des Achéens.

101. NAGY (1994), p. 377-382. Il conviendrait également de citer un quatrième exemple, moins spectaculaire mais peut-être aussi exemplaire par sa concision : il s'agit de la mention par Nestor de

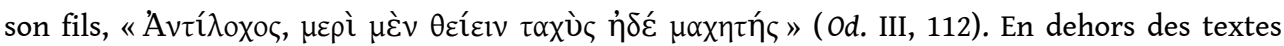
homériques, rappelons qu'Héraclès fut suffisamment bon coureur pour faire jeu égal avec la biche de Cérynie.

102. NAGY (1994), p. 371-372 et 276; DUMÉZIL (1986), p. 59-65, avec cette différence d'approche que Dumézil cherche un équivalent à la bipartition de type Vayu-Indra dans les deux figures d'Héraclès et d'Achille là où Nagy souligne le double visage du héros de l'Iliade.

103. Hom., Il. XVI, 213 ; XXIII, 713. B??, ín, ou ǐc : cf. NAGY (1994), p. 369-370.

104. Rapidité de Zéphyr : Il. XIX, 415-416; engendre Zanthos et Balios : XVI, 150-151.

105. Il. XX, 223-229. 
106. Sur les ailes des Boréades, voir Pindare, Pythique IV, 179, et Apollonios de Rhodes, I, 219220, avec le commentaire de VIAN (1974), p. 60 ; leurs victoires aux concours : schol. ad Ap. Rh. I, 1304 ; Hygin, Fab., 273, 10 ; Philstr., Gym., 3.

107. Les ailes sont à entendre avant tout comme un symbole de la rapidité et ne dénotent pas nécessairement la capacité de voler. Dans une récente étude des figures ailées sur les peintures de vases des $\mathrm{VI}^{\mathrm{e}}$ et $\mathrm{V}^{\mathrm{e}}$ siècles, Arne Thomsen arrive à la même conclusion pour le domaine iconographique : THOMSEN (2011), p. 37-56. Dans ce domaine également, la connotation l'emporte sur la dénotation.

108. Cette course consiste à courir sur le sommet, غ̇ं ' ókpov, des épis ou des vagues. L'exemple canonique est donné par les cavales filles de Borée : Hom., Il. XX, 226-229, ou par Iphiclos dans Hésiode, Catalogue fr. 62.

109. Cette idée que les cavales sont fécondés par le vent durera toute l'antiquité, au prix de légères tentatives de rationalisation : voir Aristote, Histoire des Animaux VI, 18, 4 (572a 10-17); Virgile, Géorgiques III, 272-279 ; Pline, Histoire naturelle VIII, 67, § 166 ; Varron, Économie rurale II, 1, 7 et Columelle, VI, 27, 7.

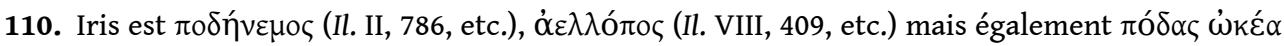

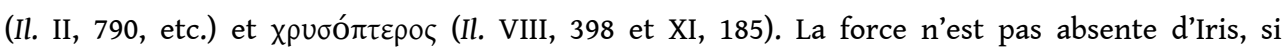
l'étymologie proposée par Gregory Nagy, qui fait dériver son nom d'un *uis ancêtre de ǐc, est exacte (NAGY [1994], p. 375 n. 6).

111. L'adjectif $\theta$ oó $\varsigma$ s'applique particulièrement aux chars, aux chevaux, à quelques guerriers, à Arès et aux navires dans les poèmes homériques. Un poème de l'Anthologie palatine (VII, 212) concentre joliment plusieurs de ces figures : on y parle d'un cheval aux pieds de vent, nommé Aî $\theta u ı$, la mouette, qui a souvent rivalisé avec les navires, et qui se retrouve comparé avec un oiseau volant sur la distance d'un dolichos!

112. Iphiclos courait sur le sommet des gerbes sans les plier: Hés., Cat. fr. 62 et schol. in Pd, $4^{\mathrm{e}}$ Pythique, 61. Apollonios de Rhodes attribuera la même qualité à Euphémos, qui pouvait courir sur le sommet des vagues : Ap. Rh. I, 179-184. Voir aussi la chasseresse et guerrière Camille : Virgile, Énéide VII, 808-811.

113. Aux bons ouvriers les bons outils : ce n'est pas un hasard si le vainqueur de la course de biges aux jeux funéraires de Patrocle est Diomède, un des rares Achéens à pouvoir prétendre au

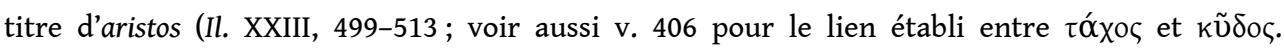
Diomède aristos : cf.NAGY [1994], p. 53-54).

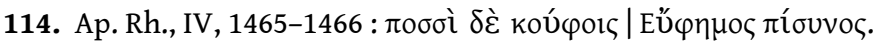

115. Les Argonautes comprennent non seulement Euphémos et Iphiclos, mais également des fratries de champions de vitesse, les Boréades et les Dioscures. Argô, façonné par Athéna ellemême, ne pouvait être que le plus rapide des navires : Ap. Rh., I, 111-114.

116. Ap. Rh., II, 269-290.

117. Voir par exemple Virgile, Énéide V, 319 ; Stace, Thébaïde VI, 558 ; 638-640 ; Silius Italicus, Guerre punique XVI, 335; 484-485; Quintus, Posthomerica IV, 516-518; 570-571; Nonnos, Dionysiaques XXXVII, $121 ; 279-283 ; 458 ; 633$ et 640-645.

118. Il. XIX, 386 (trad. P. Mazon).

119. Il. XXII, 458-459.

120. Il. XXII, 136-137 et 157-158.

121. Il. XXII, 22.

122. Il. XXII, 143 et 198.

123. Il. XXII, 157-161.

124. Il. XXII, 162-166. 
125. C'est l'occasion de rappeler que la rapidité d'Achille vaut aussi pour la chasse: selon Pindare, à l'époque de son éducation agreste par Chiron, il chassait sans chien ni filet et rattrapait à la course les cerfs ( $\mathrm{Pd}, 3^{e}$ Néméenne, v. 51-52).

126. Xén., Commandant de cavalerie III, 1 ; V, 4 ; VIII, 9 ; Art équestre VIII, 10-12 ; Cynégétique XII, 4 , etc. On y retrouvera certains thèmes familiers : pour Xénophon, la pratique de l'équitation est ce qui se rapproche le plus du fantasme humain d'avoir des ailes : Commandant de cavalerie VIII, 6.

127. Londres B240. L'image a été diversement interprétée: Psyché de Patrocle, Achille bondissant sur le rivage troyen à l'arrivée de sa nef, ou encore envol d'Achille vers l'île des bienheureux : voir RECKE (2002), p. 250.

128. [Apollodore], III, 12, 5 et épitomé III, 33 ; schol. Hom., Il. VI, 49 ; schol. Lyc., 302. Hom., Il. XXIV, 257, donne au contraire de Troilos l'image d'un guerrier adulte.

129. LIMC, I (1981), Achilleus, no 206-288 et Troilos. Voir RECKE (2002), p. 203-208.

130. Voir par exemple la description qu'en donne STRAWCZYNSKI (2006), p. 30-35.

131. Par exemple sur une péliké du Louvre, G231, ou sur le fragment d'une coupe d'Istanbul par le peintre de Pistoxenos (Musée archéologique A33.2347).

132. Chien: par exemple sur une amphore de Berlin, Pergamonmuseum F1685; le chien est l'épisème d'Achille sur une coupe du peintre de Brygos du Louvre (Louvre G154). Lièvre : coupe du peintre $C$ de New-York (Met GR 521). En tant que cadeau pédérastique, le lièvre peut également rappeler les versions qui faisaient d'Achille un éraste repoussé ; mais dans le même temps, c'est une proie habituelle de la chasse, et un animal dont Xénophon vante la rapidité : Art de la chasse V, $17 ; 27$, etc. Sur Achille chasseur, voir la note 124.

133. À quelques exceptions près : voir par exemple la coupe du peintre de la Fonderie (Boston 98.933), une des rares images à représenter clairement une poursuite.

134. Il s'agit à ce jour de seize vases recensés par le LIMC I, Achilleus, no 585-600, et plus récemment par RECKE (2002), p. 72-74.

135. Voir notamment la superbe hydrie de Boston (63.473) sur laquelle Achille porte les trois jambes du triskèle en épisème et bondit vers son char, tandis qu'une déesse ailée et l'eidôlon de Patrocle, ailé lui aussi, accourent à sa rencontre.

136. Philstr., Gym., 32.

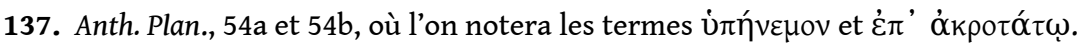

138. Paus., II, 19, 7 et III, 21, 1. Pour MUSTI - TORELli (1986), p. 275, la statue argienne est celle du second Ladas (voir infra), le champion de stade, mais outre qu'on voit mal ce qu'un Achéen ferait

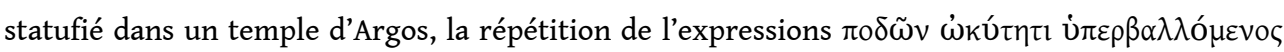

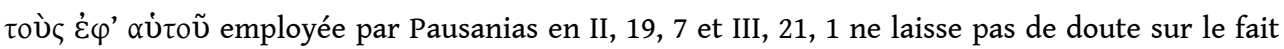
qu'il parle du même coureur. Quant à l'histoire de la mort de Ladas, il y a là, me semble-t-il, le germe d'un motif qu'on rencontrera chez d'autres coureurs, la mort au terme de la course victorieuse, qui rejoint à la fois l'idéal grec du décès en pleine gloire et le thème de la disparition mystérieuse de l'athlète, prélude à son culte. Sur ce sujet, voir par exemple BILINSKI, 1960, p. 3031 ; FONTENROSE (1968), p. 76-78 (narratème F) ; CURRIE (2005), p. 131-132.

139. Anth. Plan., 53, L'épigramme n'a été transmise que partiellement par l'anthologie et c'est en se fondant sur d'autres citations et sur des détournements de l'épigramme que Pierre Laurens a restitué les deux derniers vers du distique : LAURENS (1973), p. 91-95 et ROBERT (1989), p. 278-279.

140. Anth. Pal. XI, 86 et 119. Cf. LAURENS (1973), p. 91-95, et ROBERT (1989), p. 278-279, qui cite également Anth. Pal. XI, 208.

141. Solin, I, 98: Verum ut ad pernicitatis titulum transeamus, primam palmam velocitatis Ladas quidam adeptus est, qui ita supra cavum pulverem cursitavit, ut harenis pendentibus nulla indicia relinqueret vestigiorum. Catulle, Poésies LV, v. 15.

142. Il n'est pas toujours aisé de savoir si les nombreuses mentions de Ladas dans la littérature romaine renvoient au sprinteur ou au fondeur. Luigi Moretti prend le parti d'en attribuer la 
plupart au vainqueur du dolichos (MORETTI [1957], no 260, p. 96) en supposant que sa statue par Myron avait été transportée à Rome et se trouvait à l'origine de sa popularité. Mais l'épigramme pour le sprinteur et ses nombreuses allusions ou parodies d'époque romaine semblent au contraire affirmer que le plus célèbre des deux était bien Ladas d'Aigion (MORETTI [1957], no 535, p. 135).

143. Voir supra, n. 108 et 112, et l'épigramme de Posidippe sur la statue de Kairos : Anth. Plan., 275, 3

144. Lucilius joue précisément sur cette idée dans une autre épigramme satirique sur un coureur : Marcus était si lent que sa course dura toute la nuit, et qu'au lendemain, les employés le prirent pour une statue de pierre : Anth. Pal. XI, 85 avec le commentaire de ROBERT (1989), p. 254-259.

145. Luc., Timon, 20.

146. Luc., De la calomnie, 12.

147. Iris : LIMC V (1990), 1, s.v. Iris, p. 758. Sur Niké, voir la documentation rassemblée par LONIS (1979), p. 231-253, et surtout l'analyse de THOMSEN (2011), p. 163-241.

148. Sur cette soudaineté de l'épiphanie, voir notamment PIETTRE (1996), p. 352-355.

149. Par exemple Il. IX, 6 ; XXI, 346-347 ; XXIII, 212-215 ; Od. XII, 408.

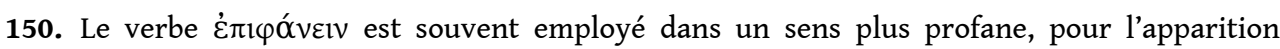
soudaine de troupes qui vont changer le cours de la bataille. Voir par exemple Xén., Hell. VII, 2, 20 ; Diod., XIV, 9, 2-3 ; XV, 36, 4.

151. Hdt., VII, 188 et 189.

152. Hdt., VII, 189.

153. CHANTRAINE (1999), p. 182-183.

154. Hdt., VII, 191. Sur le rapport d'Achille avec les tempêtes, voir NAGY (1994), p. 387-395. Thétis, la seiche (sépia en grec) et la perdition en mer : DETIENNE - VERNANT (1974), p. 161-166 ; NAGY (1994), p. 394.

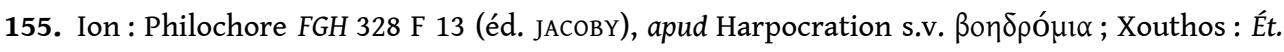

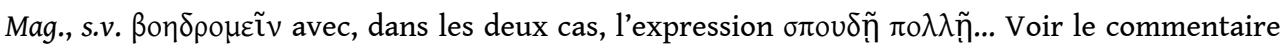
de JACOBY, p. 281, pour la place d'Apollon dans ces histoires de courses, ainsi que SALVIAT (1992), p. 266-267, sur les aspects guerriers de la fête.

156. Plut., Camille, 19, 4-6, à quoi il faut ajouter la victoire sur les amazones : Plut., Thésée, 27, 3. Sur les Nikétéria, fête qui aurait commémoré la victoire d'Athéna sur Poséidon et qui tombait le 3 $\mathrm{du}$ mois, voir PARKER (2005), p. 476-477. Sur boédromion comme mois des victoires, voir les remarques de JACOBY ad FGrHist 328 F 13, p. 281, et de LORAUX (2005), p. $182 ; 308$, n. 49 et 50.

157. Hdt., IX, 100-101.

158. Eurymédon : scholie à Eschine, I, 128 ; Mycale : Procope le Rhéteur, Lettres, 40. Dans les deux

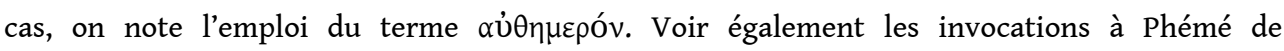
Bacchylide : Épinicies II, et $\mathrm{X}$, où la déesse vient avec Niké couronner l'athlète.

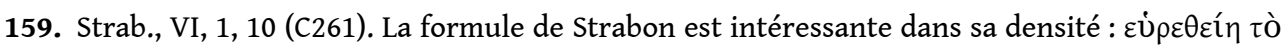

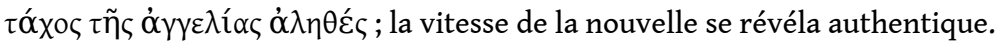

160. Paus., V, 8, 4 ; Hyg., Fab., 273, 10. On notera d'ailleurs que, sur un vase au moins, Castor est représenté ailé: THOMSEN (2011), p. 157 (le personnage est identifié avec certitude par une inscription).

161. Pline, Histoire naturelle VII, 22, § 86.

162. Julius Africanus, fr. 65 (éd. WALlRAFF), 210, apud Eusèbe, Chron. I, 206, 25 (éd. schoene); cf. MORETTI (1957), no 464, p. 127, et BILINSKI (1960), p. 25. À comparer avec l'inscription de Drymos (IG $\left.I^{2} 1,618\right)$, coureur qui a annoncé $\alpha \dot{\theta} \theta \tilde{\eta} \mu \alpha \rho$ le concours olympique aux Épidauriens. Version comique de ce thème dans Luc., Navire, 44 : le héros rêve d'avoir des ailes et de pouvoir voler

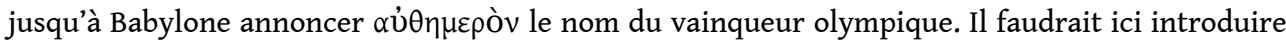
la figure de l'hémérodromos, ce coureur de fond utilisé pour transmettre des messages sur de 
longues distances, particulièrement utile dans le Péloponnèse où le relief montagneux rendait inapproprié l'emploi du cavalier. À certains d'entre eux on attribuait des performances extraordinaires, par exemple parcourir 1200 stades en un jour, qui se rapprochent de cette thématique de l'instantanéité : voir les études fondamentales de BILINSKI (1959), p. 69-80, et (1960), p. 24-26 ; ainsi que MATTHEWs (1974) ; LEE (1984).

163. Philstr., Gymn., 7.

164. Plut., Gloire Ath.,3 (Mor., 347c), et Luc., Pro Lapsu, 3. Voir BILINSKI (1960), p. 5-32 (qui défend, sûrement à tort, l'historicité de l'épisode) ; SIEWERT (1990) ; KERTESZ (1991).

165. L'euaggelia désigne un sacrifice pour une heureuse nouvelle, mais semble s'être spécifié dans l'annonce de victoires militaires - et aussi agonistiques : cf. par exemple Diod., XV, 74, 2 pour la victoire de Denys aux concours de tragédie des Lénéennes, ou Philstr., Vie d'Apollonios V, 8 pour les victoires olympiques de Néron. Il semble être à peu près synonyme des épinikia et niketeria, qu'on retrouve aussi bien en contexte militaire qu'agonistique (on notera, par exemple, que l'inscription $I G \mathrm{II}^{2}, 2311$ mentionne, après la liste des prix des vainqueurs aux Panathénées, le nombre de bêtes qu'on sacrifiera pour les niketeria des vainqueurs).

166. Plut., Aristide, 20, 4-6.

167. Sur les Eleutheria et sur leur fondation à la datation controversée, la bibliographie est vaste. Je me contente de citer ÉTIENNE - PIÉRART (1975), p. 63-75; PRANDI (1988), p. 161-179; SCHACHTER (1994), p. 136-141 ; NAFISSI (1995), p. 124-129 et VAN NIJF (2005), p. 273-277.

168. Sources et analyses dans ROBERT (1937), p. 140-142 ; (1969), p. 758-767 ; (1989), p. 323-326, et MORETTI (1953), p. 119.

169. Vaincre ou mourir : cf. ROBERT (1989), p. 334-337 ; CROWTHER (2004), p. 315-316 ; FINLEY PLEKET (2008), p. 194. Mort et kléos : VERNANT (1989), p. 41-79 et 81-84 ; NAGY (1994), p. 213-251.

170. Euklès: Plut., Gloire Ath., 3 (Mor., 347c) ; fondation du temple d'Eukleia: Paus., I, 14, 4. Euchidas enterré dans le sanctuaire d'Artémis Eukleia: Plut., Aristide, 20, 6. Voir aussi BILINSKI (1960), p. 26-28.

171. Il est vrai qu'une partie de l'historiographie récente, autour de WEES (2004), tend à reconsidérer la conception traditionnelle de la guerre hoplitique et à redonner toute leur place aux troupes légères. Hans van Wees accepte cependant l'idée qu'au v $\mathrm{v}^{\mathrm{e}}$ siècle les pratiques de la guerre accordent la prédominance à l'hoplite combattant en rangs bien compacts, et l'on notera que le discours civique privilégie continument le fantassin lourd et l'esprit de solidarité des hoplites.

172. À cet égard, je trouve très intéressante l'analyse de Stephan Müller qui tend à appliquer la même grille de lecture à la création de l'épreuve de l'apobatès : MÜLLER (1996), p. 56-69.

173. Il était impossible de courir une telle distance avec le lourd armement du début du ve siècle et de combattre ensuite. D'après les reconstitutions de DONLAN - THOMPSON (1976) et (1979), les Athéniens n'ont dû courir que le dernier stade qui les séparait de leurs adversaires. Sur la traduction religieuse de cette charge, par laquelle s'opposent course et phobos, voir surtout ELLINGER (2002), p. 322-323.

174. Hdt., VI, 116. On notera qu'Isocrate, pour sa part, déplace encore plus en amont la course victorieuse : l'exploit, pour lui, consiste à s'être rendu à Marathon à grande vitesse, le jour même où les Perses débarquèrent, et - conséquence logique de ce déplacement - à les avoir vaincus ce même jour : Isocrate, Panégyrique (IV), 86-87.

175. Plut., Pélopidas, 23, 5. Seul auteur à insister sur la vitesse de la charge, Plutarque est cohérent et explique qu'elle entraîna immédiatement la dislocation et la fuite des Spartiates. Que cette charge victorieuse soit attribuée à un bataillon d'élite est évidemment significatif. Ce n'est d'ailleurs pas le seul exemple ; Xénophon rapporte ainsi que les epilektoi thébains tentèrent un coup contre Corinthe en courant à toute vitesse : Xén., Hell. VII, 1, 18-19 (je remercie John Ma de m'avoir indiqué cette référence). 
176. Plut., Alexandre, 4,10 , et Apophthegmes, Alexandre 2 (Mor., 179d).

177. Par exemple Thuc., II, 25,2 ; IV, 79, 1 ; 104, 3 ; 111, 2 ; 124, 3. Cf. HORNBLOWER (1996), p. 38-61.

178. Thuc., IV, 121 avec le commentaire de HORNBLOWER (1996), p. 380-385.

179. Plat., Banquet, 221c.

\section{RÉSUMÉS}

Dans le débat antique sur l'utilité militaire des compétitions athlétiques, la course à pied a parfois reçu l'appui inattendu de philosophes et de soldats. Il est vrai qu'à Olympie cette épreuve était la seule à comporter une variante avec armes, et qu'on la retrouve dans quelques rites qui semblent avoir pour fonction d'accorder à des jeunes gens les titres d'adulte et de guerrier. Plus généralement, le coureur semble entouré d'une aura héroïque, parfois concrétisée en un véritable culte, dont Achille fournit le modèle idéal. Dans l'épopée, la course fonctionne en effet au sein d'un système de représentations qui associe toute une série d'incarnations de la vitesse (Achille, vents, chevaux, navires...) aux notions de force, d'instantanéité et de victoire. Ces représentations ont suffisamment modelé la pensée grecque pour rester prégnantes tout au long de l'histoire de l'athlétisme et de la guerre antiques; elles offrent ainsi une clef de lecture de nombreuses anecdotes militaires.

While athletic competitions in ancient Greece were often criticized for their lack of military utility, footraces sometimes received unexpected support from certain philosophers and soldiers. Admittedly running was the only Olympic event to include a version involving weapons, and to be performed during puberty rites in order to grant adult, warrior status to the youth of the city. More generally, the runner seems to have been surrounded by a heroic aura (sometimes inspiring a cult following), for which Achilles provides the ideal model. In epic poetry, racing belongs to a system of representations that combines various embodiments of speed (Achilles, winds, horses, ships, etc.) with notions of power, instantaneity and victory. These representations have shaped Greek thought sufficiently to remain present throughout the history of ancient athletics and war, and to offer a key for understanding many military anecdotes.

\section{AUTEUR}

\section{VINCENT CUCHE}

LabEx RESMED - Paris 